\title{
El Aprendizaje del Concepto de Campo en Física: una Investigación Exploratoria a Luz de la Teoría de Vergnaud
}

\author{
Alfonso Llancaqueo*, $\mathrm{M}^{\mathrm{a}}$ Concesa Caballero** y Marco Antonio Moreira*** \\ * Departamento de Ciencias Físicas.Universidad de La Frontera, Chile, allanca@ ufro.cl \\ ** Departamento de Física, Universidad de Burgos, España, concesa@ubu.es \\ *** Instituto de Física, Universidade Federal do Rio Grande do Sul, RS, Brasil, moreira@if.ufrgs.br
}

Recebido em 06 janeiro, 2003. Aceito em 08 de setembro, 2003.

\begin{abstract}
Se presentan resultados de una investigación del concepto de campo, con alumnos de Bachillerato y primer año de Universidad, en el marco de la teoría de campos conceptuales de Vergnaud, adoptada como referencial teórico para comprender y explicar el proceso de aprendizaje de este concepto fundamental de la física. El propósito fue explorar vínculos entre la estructura cognitiva de los estudiantes y la estructura del concepto de campo construido por la física, y describir niveles de conceptualización del concepto. Los resultados confirman potencialidades de la teoría para abordar estos vínculos, que se manifiestan en el uso de representaciones simbólicas y una explicitación parcial de significados, que dan forma a invariantes operatorios que usan para enfrentar situaciones y problemas que demandan una conceptualización científica aceptable. Se observa una ausencia notoria de invariantes para enfrentar situaciones y problemas propuestos. El 92,7\% de los estudiantes se ubica en los niveles más bajos de conceptualización del concepto de campo (niveles 1, 2 y 3 definidos en este estudio), donde el nivel 3 corresponde a un reconocimiento y explicación parcial de significados del concepto. Este bajo nivel es concordante con bajos desempeños en las categorías de análisis, de operación $(17,6 \%)$, expresión escrita $(17,3 \%)$, representación $(13,6 \%)$ y resolución $(5,5 \%)$, en acuerdo con la teoría de Vergnaud, que afirma que un bajo nivel de conceptualización se caracteriza por bajos niveles de explicitación de invariantes y sus representaciones, con predominio de aspectos procedimentales de las operaciones sobre el uso de predicados de mayor riqueza conceptual.
\end{abstract}

Palabras-clave: concepto de campo en física; aprendizaje de conceptos; campos conceptuales de Vergnaud.

Results of a study on the acquisition of the field concept in physics by high school and college freshmen students, carried out under the framework of Vergnaud's conceptual fields theory, are presented. The aims of the study were to explore the links between students' cognitive structure and the formal structure of the field concept in physics, and to describe their levels of conceptualization of such a concept. Research findings confirm the potentialities of Vergnaud's theory to understand and explain the learning process of this basic physical concept. The links would manifest themselves in the use of symbolic representations and in a partial explicitation of some meanings that would suggest the presence of some operational invariants students use to cope with situations and problems that require an acceptable scientific conceptualization. However, a clear absence of scientifically accepted operational invariants was observed. About $92 \%$ of the students were located at the lowest level of a 1 to 3 conceptualization scale defined in the study, where 3 corresponds to the recognition and partial acquisition of meanings of the field concept. This low level of conceptualization correlates with poor performance in the following categories of analysis : operational $(17,6 \%)$, written expression $(17,3 \%)$, symbolic representation $(13,6 \%)$, and problem solving $(5,5 \%)$. These results agree with Vergnaud's theory that states that a low level of conceptualization corresponds to lower levels of explicitation of operational invariants and their representations, and predomination of procedural aspects over conceptually richer predicates.

Keywords: field concept in physics, concept learning, Vergnaud's theory of conceptual fields.

\section{Introducción}

El concepto de campo es fundamental en física por cruzar amplios dominios de la disciplina. Se ancla en el concepto de función y se utiliza para describir el comportamiento de magnitudes que se definen en todo punto de una región del espacio y del tiempo. En física clásica, este concepto es fundamental para describir y explicar fenómenos electromagnéticos, gravitacionales y de transporte, y en la física contemporánea en las teorías de partículas elementales que buscan la elaboración de modelos que expliquen y unifiquen las fuerzas básicas de la naturaleza (Feyman, 1985), lo mismo en la teoría de la relatividad general donde el

\footnotetext{
* Doctorando del Programa Internacional de Doctorado en Enseñanza de las Ciencias de la Universidad de Burgos, España, en convenio con la Universidad Federal de Rio Grande do Sul (Porto Alegre, Brasil)
} 
concepto de campo es clave para describir lo físicamente real (Einstein, 1995), o sea un campo son ciertas cualidades físicas de los puntos del espacio y el tiempo (Pauli, 1996).

En la histórica de la física, el concepto de campo surge en el siglo XIX, como una búsqueda de explicación de los fenómenos electromagnéticos, aunque a finales del siglo XVIII se conocía una formulación de la gravitación en términos de campos de fuerza y de potencial, desarrollada por Laplace y Poisson, esta parecía un "dispositivo matemático" más que una formulación que permitiese una profundización conceptual física (Slater y Frank, 1947). Faraday, fue quién inició un abordaje teórico basado en el concepto de campo, que rechazaba la idea de acción a distancia para las fuerzas electromagnéticas. Sin embargo, fue Maxwell en 1855, quién da un cuerpo matemático a las concepciones de Faraday, elaborando un programa de investigación basado en el supuesto ontológico: que una acción electromagnética, se transmite continuamente por el espacio y tiempo mediatizada por el campo, y no se ejerce a distancia según era el supuesto ontológico de la mecánica de Newton que dominaba la imagen de naturaleza de esa época (Berkson, 1981; Harman, 1990).

Maxwell desarrolla y consolida un movimiento metodológico y una actitud epistemológica para la investigación basado en el uso conjunto de tres métodos: la analogía entre dominios de la física, el método hipotético deductivo y el analítico, consolidando sus investigaciones en 1868 con la formulación de la teoría de los campos eléctricos y magnéticos expresada en cuatro ecuaciones para dichos campos. Esta teoría es la primera donde la idea de campo adquiere significación física y se funda en la afirmación que una carga eléctrica está rodeada por un campo eléctrico de alcance infinito, y que el movimiento de una carga eléctrica origina un campo magnético también de alcance infinito. Ambos campos son magnitudes vectoriales definidas en cada punto del espacio y el tiempo. La teoría electromagnética fue construida sobre las ideas de espacio y tiempo de la mecánica que describe la interacción entre partículas mediante el concepto de fuerza o de energía potencial de interacción que es una función de las coordenadas espaciales de las partículas en interacción, suponiendo una propagación instantánea de las mismas. Así, las fuerzas sólo dependen de la posición de las partículas en cada instante de tiempo, de modo que un cambio de posición de una partícula en interacción afecta inmediatamente a las otras. No obstante, Maxwell encuentra que para las interacciones electromagnéticas existe una velocidad límite de propagación, la velocidad de la luz. Por lo tanto, una mecánica basada en el supuesto de una propagación instantánea para las interacciones parecía no ser correcta.

En la teoría de la relatividad, Einstein por el contrario, parte de la idea que si sobre una partícula en interacción ocurre un cambio, éste influirá sobre los otras después de transcurrido un intervalo de tiempo, luego existe una velocidad de propagación de la interacción que determina el tiempo transcurrido desde el momento en que una partícula experimenta un cambio y comienza a manifestarse en otra (Landau, 1992; Slater y Frank, 1947). De este principio se sigue que la velocidad de propagación para las interacciones es igual en todos los sistemas de referencia inerciales (aquellos donde se cumple el principio de inercia de Newton) e igual a $\mathrm{c}=2,99793 \times 10^{8} \mathrm{~m} / \mathrm{s}$, que es la velocidad de propagación de la luz. En consecuencia, la teoría de la relatividad toma la idea que la interacción entre partículas se puede describir mediante el concepto de campo de fuerzas, afirmando que una partícula crea un campo en torno de ella, entonces una fuerza actúa sobre cada partícula situada en ese campo. En la mecánica clásica, el campo es un modo de describir un fenómeno físico, en cambio en la teoría de la relatividad, debido al valor finito de la velocidad de propagación de las interacciones, las fuerzas que actúan sobre una partícula en un instante no están determinadas por las posiciones de las otras en el mismo instante, ya que un cambio en la posición de una de las partícula afecta a las demás después de transcurrido un cierto tiempo. Esto hace que el campo adquiera realidad física, luego, no se puede hablar de una interacción directa entre partículas colocadas a una cierta distancia, sino de la interacción de una partícula con el campo y de la interacción posterior del campo con otra partícula (Landau, 1992; Einstein, 1995).

Por otra parte, en la psicología cognitiva, sobre el estudio de los conceptos, se han realizado investigaciones, para determinar qué son, cómo se representan y para qué sirven. En general, los conceptos se entienden como representaciones mentales de clases (de situaciones, objetos, eventos, individuos) que incluyen información de las instancias de la clase más información adicional que se relaciona con la definición de la clase en relación con otros conceptos, más información procedente de la percepción, de la vivencia de experiencias, de las inferencias realizadas (Rodríguez, 1999).

Las investigaciones realizadas en psicología, han dado origen a diversas teorías sobre los conceptos y modelos de representación conceptual, que se extienden entre otros, desde la teoría clásica de los conceptos, con raíces en la filosofía de Platón, la teoría de prototipos, la teoría de la teoría, la teoría neoclásicas y el atomismo conceptual (Laurence y Margolis, 1999; Lakoff, 1999; Medin, 1998), las cuales ponen de manifiesto que las personas no utilizan un único tipo de representación, sino distintos tipos de representación conceptual en función de los significados del concepto, del contexto y su nivel de conocimientos (Rodríguez, 1999). Por otra parte, en las teorías de desarrollo cognitivo constructivistas, como la de Piaget, las unidades psicológicas del funcionamiento del sujeto son los esquemas, los cuales son una parte esencial de las representaciones, pero éstas, también están constituidas por otros elementos, como los conceptos, las relaciones entre éstos y su organización en teorías, que corresponden a un tipo de representaciones más elaboradas y explícitas (Delval, 1997). De este modo, los conceptos se generan a partir de la aplicación de los esquemas y describen las regularidades que un sujeto encuentra al usarlos, constituyendo un instrumento esencial del conocimiento que contribuye a la economía cognitiva de los sujetos.

En la actualidad, en la investigación en educación en ciencias, se considera importante conocer cómo los estudian- 
tes construyen los conceptos científicos, qué tipo de representaciones construyen, qué procesos cognitivos ocurren, y cómo asimilan sus significados, ya que esto permitiría conocer el desarrollo conceptual como una construcción y discriminación de significados (Moreira, 2000) y guiar el diseño de modelos de enseñanza para una mejor comprensión del conocimiento científico. Para los fines de la educación en ciencias, entendida como hacer que los estudiantes compartan los significados de las ciencias, en el sentido de interpretar el mundo desde el punto de vista de las teorías científicas, generando nuevas capacidades representacionales que hagan posibles nuevas formas de conocimiento, que les alejen de la inmediatez de los conocimientos intuitivos (Moreira, 1998; Pozo y Gómez Crespo, 1998; Pozo, 2002), un aprendizaje significativo de conceptos claves, como el concepto de campo, es una condición necesaria para la formación científica de los estudiantes, su comprensión de los fenómenos físicos y conocimiento de principios que sustentan aplicaciones tecnológicas.

Las investigaciones en educación en ciencias sobre el concepto de campo se han realizado principalmente, con estudiantes secundarios y primer nivel de universidad sobre el campo electromagnético y gravitatorio. Una revisión de éstas se encuentra en una publicación anterior (Llancaqueo, Caballero y Moreira, 2003), donde las aportaciones se clasifican en cuatro categorías: estrategias de enseñanza; concepciones y razonamiento, representaciones mentales y dificultades de aprendizaje. Un resumen de sus principales características y tendencias se presenta en la Tabla 1.

Respecto a enfoques teóricos del aprendizaje, formas de representación y desarrollo conceptual, una minoría de estas investigaciones trata estos aspectos. La mayoría utiliza como referentes, el enfoque de concepciones alternativas, o un modelo de formas de razonamiento común en física, o un modelo de enseñanza-aprendizaje como una investigación dirigida. Las dificultades de aprendizaje se caracterizan como problemas de enseñanza y epistemológicos, similares a los surgidos en los orígenes del concepto de campo (Furió y Guisasola, 1998a; 1998b; Furió y Guisasola, 2001; Martín y Solbes, 2001), abordándose desde el modelo de enseñanza-aprendizaje como investigación orientada, por la ventaja de articular los aspectos conceptuales, procedimentales y actitudinales del aprendizaje del conocimiento científico (Gil, 1985; Gil y Carrascosa, 1993; Furió 2001), frente al enfoque de las concepciones alternativas, que se centra, en la adquisición del conocimiento y el cambio conceptual (Driver, R., Guesne, E. y Tiberghien, A., 1985; Marín, 1999; Di Sessa y Sherin, 1998; Pozo, 1999).

Otro aspecto importante de considerar, es la complejidad y amplitud de los significados físicos y matemáticos del concepto de campo, percibiéndose la necesidad de explorar un referencial teórico que permita abordar los vínculos entre la estructura del conocimiento del concepto construido por la física, y la estructura conceptual de los estudiantes del mismo, (Vergnaud et al., 1981; Pozo, 1996).

Todo esto destaca la importancia del aprendizaje del concepto de campo en los estudiantes que no se restringe a los niveles universitarios, sino que existe interés en introducirlo desde niveles educativos anteriores a la universidad, ya que su aprendizaje crearía la posibilidad de aprendizaje de otros conceptos específicos de la física, integrando a éstos en una estructura conceptual más general, como lo es el concepto de campo, de modo que la comprensión de conceptos científicos específicos (por ejemplo, temperatura, presión, otros) se vería facilitada si los estudiantes aprenden a analizar problemas y situaciones del mundo natural desde la estructura conceptual de campo.

En el presente trabajo, se presentan resultados de una investigación exploratoria sobre el concepto de campo, con alumnos de Bachillerato y primer año de Universidad, usando la teoría de campos conceptuales de Vergnaud, adoptada como referencial para comprender y explicar el aprendizaje significativo del concepto.

\section{Fundamentación teórica}

\subsection{La teoría de los campos conceptuales de Vergnaud}

Para Gérard Vergnaud, al igual que para Piaget, el conocimiento es un proceso de adaptación. Sin embargo, para Vergnaud, el problema central de la cognición es la conceptualización, y a partir de esta premisa desarrolla una teoría psicológica que postula que el conocimiento se encuentra organizado en campos conceptuales de los cuales los sujetos se apropian a lo largo del tiempo. Los campos conceptuales se definen como grandes conjuntos informales y heterogéneos de situaciones y problemas, en que para su análisis y tratamiento son necesarios diversas clases de conceptos, representaciones simbólicas, operaciones del pensamiento y procedimientos que se conectan unas con otras durante su aprendizaje o adquisición (Vergnaud, 1983; 1990). El objetivo de la teoría es propiciar una estructura para la investigación sobre actividades cognitivas complejas, en especial el aprendizaje del conocimiento científico, “... se trata de una teoría psicológica del conocimiento o de la conceptualización de lo real que permite estudiar las filiaciones y rupturas entre conocimientos desde el punto de vista de su contenido conceptual..." (Vergnaud, 1993 p.1). Esta teoría permite analizar la relación entre los conceptos en su dimensión de conocimientos explícitos y los invariantes operatorios implícitos del comportamiento de los sujetos en determinadas situaciones, así como profundizar el análisis de las relaciones entre los significados y significantes de un concepto o de un campo conceptual (Vergnaud, 1993). Para una comprensión de la teoría de los campos conceptuales describiremos a continuación los principales significados de los conceptos claves de esta teoría.

Una situación es entendida como una tarea y toda situación compleja es una combinación de tareas y problemas, de modo que los procesos cognitivos y las respuestas 
Tabla $\mathrm{N}^{o}$ 1. Características de publicaciones de investigaciones educativas sobre el concepto de campo.

\begin{tabular}{|c|c|}
\hline $\begin{array}{l}\text { Estrategias } \\
\text { enseñanza }\end{array}$ & $\begin{array}{l}\text { Estas investigaciones tienen por propósito el diseño y evaluación de estrategias de enseñanza del campo } \\
\text { eléctrico para estudiantes secundarios de bachillerato, con propuestas sustentadas en un modelo de } \\
\text { aprendizaje como una investigación dirigida (Furió y Guisasola, 2001; Martín y Solbes, 2001) orien- } \\
\text { tado a superar dificultades de aprendizaje, asumidas a partir de resultados de investigaciones anteriores } \\
\text { sobre concepciones alternativas, y resultados de análisis de contenidos y de formas de razonamiento } \\
\text { (Viennot y Rainson, 1999). Del análisis de los resultados de la aplicación de las estrategias y de la eva- } \\
\text { luación de aprendizaje conceptual, se observa que la mayoría de los estudiantes mejora su aprendizaje } \\
\text { del concepto de campo e informan de una mayor asimilación de las ideas significativas del concepto } \\
\text { frente al grupo control. Las dificultades de aprendizaje se interpretan como consecuencia de un tra- } \\
\text { tamiento didáctico deficiente y confuso (Martín y Solbes, 2001). Además, los resultados destacan, la } \\
\text { detección de dificultades de los estudiantes para aceptar la existencia de un campo eléctrico en un me- } \\
\text { dio donde las cargas están inmóviles, e ignorar las fuentes de campo no representadas explícitamente } \\
\text { en forma matemática (Viennot y Rainson, 1999). Se concluye que la comprensión conceptual de los } \\
\text { estudiantes progresa sólo cuando los aspectos causales han sido enfatizados durante la enseñanza. }\end{array}$ \\
\hline $\begin{array}{l}\text { Dificultades } \\
\text { aprendizaje }\end{array}$ & $\begin{array}{l}\text { Estas investigaciones tienen por objetivo principal identificar, interpretar y analizar dificultades de } \\
\text { aprendizaje del campo electromagnético en contextos de enseñanza con estudiantes secundarios (15-17 } \\
\text { años) y primer año de universidad (17-18 años), bajo la hipótesis de que éstas dificultades se originan } \\
\text { y relacionan con las concepciones alternativas de la mecánica (Galili,1995), o bien se deben a un para- } \\
\text { lelismo entre los problemas de aprendizaje y los problemas epistemológicos (Furió y Guisasola, 1998) } \\
\text { que hubo que superar en la historia del desarrollo del electromagnetismo. Los resultados y conclusiones } \\
\text { de este grupo de investigaciones muestran que una minoría de los estudiantes de enseñanza secundaria } \\
\text { y universitaria usan en forma significativa el concepto de campo. La mayoría no establece diferencias } \\
\text { conceptuales entre fuerza y campo eléctrico, y se aprecia que la introducción del concepto de campo } \\
\text { a partir de su definición operacional afecta su comprensión, y a su vez, hace evidentes problemas de } \\
\text { aprendizaje de la mecánica. Se infiere la confirmación de la hipótesis que el origen de las dificultades } \\
\text { de aprendizaje podría estar en las concepciones alternativas y en un paralelismo entre problemas de } \\
\text { aprendizaje y problemas epistemológicos históricos de los orígenes del concepto de campo eléctrico. }\end{array}$ \\
\hline $\begin{array}{l}\text { Representaciones } \\
\text { mentales }\end{array}$ & $\begin{array}{l}\text { Estas investigaciones están orientadas a identificar los modelos mentales (Johnson-Laird,1983) que } \\
\text { usan las personas para pensar acerca del magnetismo y las relaciones entre electricidad y magnetismo } \\
\text { (Borges y Gilbert, 1998), e investigar el tipo y nivel de representación mental del concepto campo } \\
\text { electromagnético que construyen y utilizan los estudiantes cuando estudian, responden preguntas y } \\
\text { resuelven problemas (Greca y Moreira, 1997; 1998), y una revisión en profundidad de la teoría de } \\
\text { representaciones mentales (Greca y Moreira, 2000), que sirve de marco teórico y orienta la identifi- } \\
\text { cación de las representaciones del campo electromagnético. Los resultados y conclusiones de éstas } \\
\text { investigaciones destacan, que las personas construyen modelos mentales simples en acuerdo con sus } \\
\text { conocimientos del mundo físico, y que la expansión del conocimiento en un dominio es por asimilación } \\
\text { y acomodación del nuevo conocimiento en modelos más sofisticados. En el caso del magnetismo, los } \\
\text { diferentes modelos de magnetismo construidos por los estudiantes son una evidencia de los efectos de } \\
\text { la instrucción (Borges y Gilbert, 1998) recibida. Por otra parte los trabajos de Greca y Moreira desta- } \\
\text { can que los estudiantes que evidencian comprensión y aplicación de los significados del concepto de } \\
\text { campo son aquellos que desarrollan un modelo mental físico del concepto según la teoría de los mo- } \\
\text { delos mentales de Johnson-Laird (1983), por tanto, aprender el marco conceptual de una teoría física } \\
\text { implicaría generar un modelo o representación mental del mismo. }\end{array}$ \\
\hline $\begin{array}{l}\text { Concepciones y razo- } \\
\text { namiento }\end{array}$ & $\begin{array}{l}\text { Este grupo de estudios apunta a investigar concepciones y formas de razonamiento de los estudiantes. } \\
\text { En particular, identificar ideas acerca de aspectos del campo eléctrico que revelen posibles obstáculos } \\
\text { de aprendizaje (Viennot y Rainson, 1992); identificar la coexistencia de concepciones alternativas y } \\
\text { concepciones aceptables científicamente del campo gravitatorio (Palmer, 2002) e indagar las relacio- } \\
\text { nes entre éstas concepciones. En el caso del campo eléctrico, los resultados de estas investigaciones } \\
\text { muestran que las explicaciones de los estudiantes evidencian un razonamiento causal lineal, que dota a } \\
\text { la carga encerrada por una superficie gaussiana de un tipo de causalidad exclusiva (Viennot y Rainson, } \\
\text { 1992) que los lleva a negar y no usar el principio de superposición del campo eléctrico. La investigación } \\
\text { referida al campo gravitatorio destaca como resultado relevante la identificación de concepciones alter- } \\
\text { nativas en igual proporción en todos los grupos de estudiantes, sin importar aparentemente la diferencia } \\
\text { de edad y los niveles de instrucción en fítsica (Palmer, 2002), lo cual implicaría formas de pensamiento } \\
\text { común que sobreviven a la enseñanza. Además, se confirma la coexistencia de concepciones alternati- } \\
\text { vas y concepciones aceptables científicamente (Palmer, 2002) enlazadas por procesos de razonamiento } \\
\text { condicional, influenciadas por el contexto de las situaciones de las preguntas. }\end{array}$ \\
\hline
\end{tabular}


cognitivas de un sujeto están determinadas por las situaciones que enfrenta, luego, es a través de su acción y el dominio progresivo de las situaciones como un sujeto adquiere los campos conceptuales que modelan su conocimiento (Vergnaud, 1990). Desde un punto de vista cognitivo, el sentido que un sujeto atribuye a una situación es el resultado de la interacción entre la situación y la representación que el sujeto hace de la misma. Según Vergnaud, el vínculo entre el comportamiento del sujeto y la representación está descrito por el concepto de esquema introducido por Piaget, el cual, se define como “...una organización invariante de la conducta para una clase de situaciones determinada...” Vergnaud (1996). Los esquemas se tienen que poner en relación, por necesidades del análisis con las características de las situaciones a las cuales se aplican. La organización de un esquema se basa en cuatro clases de elementos principales: 1) Objetivos y anticipaciones; 2) Reglas de acción, de acopio y de control de la información; 3) Invariantes operatorios y 4) Posibilidades de inferencia (Vergnaud, 1996). Por objetivos y anticipaciones se entiende que un esquema se dirige siempre a una clase de situaciones en las que el sujeto puede descubrir un posible propósito de su actividad, sus objetivos, o bien esperar ciertos efectos o fenómenos. Las reglas de acción son la parte generadora de los esquemas, y permiten generar la continuación de las acciones de transformación de lo real, de la recogida de información y del control de los resultados de la acción. Son reglas del tipo si,..., entonces, que permiten al sujeto garantizar el éxito de su actividad en un contexto que puede estar en permanente cambio. Los invariantes operatorios constituyen la base conceptual implícita, o explícita, que permite obtener la información pertinente e inferir de ella, a partir de esta información y del propósito por alcanzar, las reglas de acción más apropiadas. Se distinguen dos categorías principales de invariantes operatorios: los conceptos-en-acción y teoremas-en-acción que se explicaran más adelante. Las posibilidades de inferencia son los razonamientos o posibilidades de inferencia que contiene necesariamente un esquema para anticiparse a una situación concreta, es decir, un esquema es un instrumento de adaptación de la actividad y de la conducta a los valores tomados por los diferentes parámetros en una situación particular.

\subsubsection{Concepto-en-acción, teorema-en-acción}

El interés central del concepto de esquema es establecer el vínculo teórico entre la conducta y la representación, y los invariantes operatorios son los que establecen esta articulación esencial, ya que la percepción, la búsqueda y selección de la información queda determinada completamente por los conceptos-en-acción disponibles en el sujeto (objetos, atributos, relaciones, condiciones, circunstancias, etc.), y por los teoremas-en-acción subyacentes en su conducta, "Un teorema-en-acción es una proposición considerada como verdadera sobre lo real, y un conceptos-enacción es una categoría de pensamiento considerada como pertinente" (Vergnaud, 1996, p.202).

\subsubsection{Conceptos}

Respecto de los Conceptos, la teoría de Vergnaud considera que los conceptos están constituidos por elementos que se relacionan. Estos elementos corresponden a un conjunto de situaciones, invariantes operatorios y sus propiedades que se expresan por medio de diferentes representaciones simbólicas. Estas consideraciones, llevan a definir un concepto como un triplete de conjuntos (Vergnaud, 1993; 1998). $C=\{S, I ; R\}$ donde, $S$ : conjunto de situaciones que dan sentido al concepto. Son el referente de un concepto; $I$ : conjunto de invariantes operatorios en que se basa la operacionalidad de los esquemas. Son el significado del concepto; $R$ : conjunto de formas de lenguaje que permiten representar simbólicamente un concepto, o sea, los invariantes operatorios, sus propiedades, las situaciones y los procedimientos de enfrentamiento con ellas. Estas representaciones son el significante de un concepto.

En términos psicológicos, el conjunto de situaciones $\mathrm{S}$ corresponde a la realidad, y los conjuntos I y $\mathrm{R}$ su representación considerada como dos aspectos del pensamiento, el significado y su significante. Para Vergnaud (1998), un concepto va adquiriendo sentido para un sujeto a través de su interacción con situaciones y problemas, ya que de esta manera podrá asimilar las propiedades que formaran sus conceptos-en-acción y teoremas-en-acción, o en forma resumida sus conocimientos-en-acción, que en la medida que sean expresados en forma explicita mediante sus significantes, esos invariantes o conocimientos-en-acción pasan a conformar el concepto de un individuo.

\subsection{Campo conceptual del concepto científico de campo}

Según una interpretación de la teoría de Vergnaud, el campo conceptual del concepto científico de campo, construido a partir de la física tendría por elementos: $C_{\text {campo }}=$ $\{S, I ; R\}$.

Donde, $S=\{F F\}$ : Es un conjunto de situaciones, que incluye fenómenos y problemas físicos $(F F)$ que describen y dan sentido al concepto de campo en algún dominio de la física, como la mecánica, electromagnetismo, relatividad, otros. Este conjunto de fenómenos y problemas físicos FF corresponde por ejemplo, en el caso de la mecánica, a situaciones que se refieren a las relaciones entre fuerzas conservativas y energía potencial, en electromagnetismo, gravitación y relatividad a situaciones y problemas relacionados con el fenómeno de interacción entre partículas y campo, o en el caso de mecánica de fluidos y conducción del calor, a situaciones que describen el comportamiento de magnitudes como la velocidad, densidad de corriente, flujo de calor u otras que se distribuyen y evolucionan en un medio continuo.

$I=\{I(F F) \cup I(O M)\}$ : Es un conjunto de invariantes operatorios físicos $I(F F)$ y matemáticos $I(O M$, científicamente aceptados, que se aplican a las situaciones, es decir, se aplican tanto a los problemas y fenómenos físicos $F F$ como a los objetos matemáticos $O M$, y mediante sus propiedades, relaciones y transformaciones dan significado al concepto de campo en cada una de las situaciones y problemas. El conjunto de invariantes físicos $I(F F)$ tiene por 
elementos, operaciones y sus propiedades, que se relacionan con las magnitudes físicas de posición, velocidad, aceleración, masa, fuerza y carga, más un conjunto de magnitudes y propiedades que se derivan o relacionan de éstas, tales como trabajo, energía, campo eléctrico, campo magnético, potencial, flujo magnético, circulación, otras.

Las propiedades de las operaciones físicas: son los principios y leyes físicas expresadas en términos de las operaciones y propiedades de los objetos matemáticos, tales como en el caso de la mecánica clásica, los Principios de Newton para el movimiento, es decir, los principios de inercia, de acción y reacción, ecuación de movimiento y principio de superposición de fuerzas, además, los teoremas de impulso y momentum, de trabajo y energía, las relaciones entre fuerza y energía potencial y las leyes de conservación del momentum lineal, momentum angular y energía mecánica. En el caso del electromagnetismo, son las leyes de Gauss, Faraday y Ampère, o sea, las ecuaciones de Maxwell, además, las ecuaciones de continuidad para la conservación de la carga y las leyes de conservación de la energía asociada al campo electromagnético.

El conjunto de invariantes matemáticos $I(O M)$ tiene por elementos los significados de los conceptos de escalar, vector, tensor, función, más las operaciones y propiedades del álgebra vectorial y las operaciones y propiedades del cálculo diferencial e integral, donde: Las operaciones del álgebra vectorial son: adición, producto entre un escalar y un vector, producto escalar y producto vectorial entre vectores. Las propiedades del álgebra vectorial, se refieren a las propiedades de las operaciones que definen un espacio vectorial, o sea, para la adición, las propiedades: conmutativa, asociativa, elemento identidad y elemento inverso; y para el producto entre un escalar y vector: las propiedades: asociativa respecto a un producto de escalares, distributiva respecto a la suma de vectores, distributiva respecto a una suma de escalares y elemento identidad. Las principales operaciones del cálculo son: Límite, derivada, integral de funciones escalares y vectoriales, diferencial exacta, gradiente de un campo escalar, divergencia, rotor, circulación y flujo de un campo vectorial. Las principales propiedades del cálculo se refieren a los teoremas de continuidad, las relaciones entre campos conservativos y potencial, y los teoremas de la divergencia y del rotor.

$R=\{R[F F \cup I(F F) \cup I(O M)]\}$ : Es un conjunto de representaciones simbólicas y pictóricas usadas en las situaciones, es decir, representaciones usadas en los problemas y fenómenos físicos $F F$, más las representaciones simbólicas de los invariantes operatorios físicos $I(F F)$ y matemáticos $I(O M)$, o sea, las representaciones de los principios y leyes físicas, y de las operaciones matemáticas y sus propiedades. Las principales representaciones de los fenómenos físicos $F F$ y de los invariantes físicos $I(F F)$ y matemáticos $I(O M)$, son las representaciones geométricas de flechas y representaciones analíticas de componentes del álgebra vectorial. Además, las representaciones proposicionales de ecuaciones, gráficas y pictóricas tales como tablas, gráficos, diagramas de flechas, líneas de fuerza, superficies de nivel de los conceptos de función, campo escalar y campo vectorial, tales como campos de temperatura, campos de fu- erza, campo eléctrico, campo magnético usados en las situaciones.

Todas estas representaciones simbólicas y pictóricas corresponden a los significantes del campo conceptual del concepto de campo construidos desde la física y matemática que facilitan hacer la distinción fundamental entre significante y significado, que es de la mayor importancia para el aprendizaje de conocimientos científicos (Vergnaud et. al., 1981), ya que los invariantes operatorios de los estudiantes se sitúan en el plano de los significados del concepto de campo, pero estos significados de los estudiantes, que no necesariamente coinciden con los significados científicamente aceptados se manifiestan en el pensamiento y en la comunicación con una explicitación simbólica o pictórica parcial mediante imágenes, dibujos, álgebra, cálculo, lenguaje natural.

De este modo, el contenido de posibles aspectos a indagar dispuestos según el triplete de conjuntos $C=$ $\{S, I, R\}$ es: Situaciones y problemas que den sentido al concepto de campo. Por ejemplo en electromagnetismo, gravitación y relatividad, situaciones y problemas relacionados con la interacción entre partícula y campo, y en el caso de mecánica de fluidos, conducción del calor, situaciones para describir el comportamiento de magnitudes en un medio continuo. Invariantes operatorios de los esquemas que dan significado al concepto, es decir, invariantes que describen un campo como una función escalar o vectorial definida en todo punto de una región del espacio y el tiempo; invariantes de las magnitudes físicas que se describen como campos, e invariantes que describen las propiedades y leyes físicas que cumplen dichas magnitudes. Representaciones simbólicas, referidas a formas de lenguaje que permiten representar las situaciones, problemas e invariantes operatorios del concepto de campo, tales como, representaciones simbólicas gráficas, lingüísticas, pictóricas y analíticas de magnitudes que se describen como campo.

En consecuencia, esta teoría podría ser aplicada para guiar investigaciones sobre el aprendizaje de conceptos de física (Moreira, 2002; Greca y Moreira, 2002), si se identifican situaciones que den sentido a un concepto, para luego investigar los invariantes operatorios usados por los estudiantes que les permiten comprender y explicar las representaciones simbólicas que ayudan a la conceptualización y asimilación de los significados del concepto.

\section{Estudio exploratorio sobre el aprendizaje del concepto de campo en física}

Considerando los antecedentes teóricos de la teoría de campos conceptuales de Vergnaud y empíricos de las investigaciones revisadas sobre el aprendizaje del concepto de campo, este estudio tiene por objetivos:

Relacionar la estructura formal del conocimiento del concepto de campo y la estructura conceptual que poseen los estudiantes respecto del mismo, utilizando como marco de referencia la Teoría de los campos conceptuales de Verg- 
naud.

Describir niveles de conceptualización de los estudiantes de Bachillerato y primer nivel de Universidad del campo conceptual del concepto de campo en física.

La hipótesis general del estudio es cuando un estudiante se enfrenta a un referente con situaciones y objetos que dan sentido al concepto de campo, esa información entra en interacción con la estructura de conocimientos-en-acción (invariantes) de los esquemas que cada estudiante dispone. Por consiguiente, sus respuestas serán una aproximación a su estructura conceptual y variarán de acuerdo con el nivel de desarrollo conceptual de cada estudiante poniéndose de manifiesto aspectos de su comprensión del concepto de campo, lo cual posibilita identificar significados del concepto de campo ya sean, científicamente correctos, alternativos o ausencia de ellos.

Esta hipótesis incluye los siguientes supuestos:

Aparecerán respuestas sobre el concepto de campo, y de conceptos del campo conceptual del concepto de campo tales como escalar, vector, función, etc. con una estructura de representaciones simbólicas y pictóricas que dan forma a los invariantes operatorios que utilizan los estudiantes y que representan significados que ellos atribuyen al concepto de campo en acuerdo o desacuerdo a los significados científicamente correctos.

La información proporcionada sobre el concepto de campo durante el proceso de enseñanza no induce de modo automático a una comprensión del concepto, sin que haya ocurrido una interacción entre esa información y la estructura conceptual de cada estudiante. Esta interacción se produce de modo diferente, según los invariantes operatorios de los esquemas que un estudiante dispone en cada momento de su desarrollo del campo conceptual del concepto de campo.

\section{Metodología}

\subsection{Sujetos}

La muestra estuvo conformada por un total de 55 sujetos, 35 chicos y 20 chicas, de entre 16 y 20 años de edad, estudiantes de la ciudad de Burgos, España; 48 cursaban Bachillerato y 7 cursaban el primer curso de la carrera Licenciatura en Química.

\subsection{Instrumento}

Considerando que no se contaba con un instrumento para obtener datos que permitieran analizar y evaluar el nivel de conceptualización del concepto de campo en el sentido descrito por la Teoría de Campos Conceptuales de Vergnaud, se procedió a la elaboración de un instrumento ad-hoc para la realización de esta investigación. Para ello se diseñaron una serie de situaciones con tareas y problemas de lápiz y papel, de las cuales se seleccionaron 10 para la construcción definitiva del instrumento (ver anexo 1).

En el instrumento se presentan situaciones con un formato diverso con preguntas de respuesta abierta, de modo que los datos obtenidos permitan inferir invariantes operatorios usados por los estudiantes para dar cuenta de las situaciones y apropiarse de representaciones simbólicas y pictóricas que les ayudan a la conceptualización y asimilación de los significados del campo conceptual del concepto de campo. Los contenidos de las situaciones y representaciones simbólicas y pictóricas incluidas en el instrumento se distribuyen a lo largo de las 10 situaciones seleccionadas que se presentan resumidos en la Tabla 2.

El propósito del instrumento es disponer de un referente con situaciones y objetos (Vergnaud, 1998) escrito en el lenguaje natural y de las representaciones simbólicas de la física, que den sentido al concepto de campo, de manera que el estudiante a través de su interacción con las situaciones y objetos, manifieste también mediante su lenguaje escrito o el lenguaje simbólico de la física, las propiedades, relaciones $\mathrm{y}$ transformaciones que dan forma a los invariantes operatorios que utiliza y que representan para él los significados que atribuye al concepto de campo.

\subsection{Análisis de la confiabilidad del instru- mento}

La validez de contenido se resguardó analizando los contenidos de las situaciones y los presupuestos de la teoría (Silveira, 1981), sometiendo el instrumento al juicio de un experto. Con el fin de investigar la calidad de los puntajes totales obtenidos en la aplicación del instrumento se realizó un análisis de consistencia interna (Silveira, 1993) de los puntajes mediante el coeficiente alfa de Cronbach, que mide la parte estable, confiable, común a los items de un instrumento (Cronbach, 1967; Silveira, 1993). Los resultados del análisis de confiabilidad para los puntajes generados en la aplicación del instrumento se presentan en la Tabla 3.

Por otro lado, como parte del análisis de consistencia interna del instrumento se investigó la posibilidad de eliminar algún item para mejorar el coeficiente alfa (Silveira, 1993), con este fin se calcularon los coeficientes de correlación de Pearson entre el puntaje de cada item y el puntaje total (ver Tabla 4).

Los valores de todos los coeficientes de correlación son positivos, igual o superiores a 0,60 , por lo tanto, la eliminación de cualquier item generaría una disminución del coeficiente alfa. Por lo tanto, si bien el valor 0,71 del coeficiente alfa implica un nivel intermedio de confiabilidad, y dado que lo se intenta medir son aspectos de los esquemas de los estudiantes, largamente implícitos, se estima que el valor obtenido del coeficiente alfa indica que los puntajes generados en la aplicación del instrumento son estadísticamente confiables.

\subsection{Procedimiento}

Se estableció contacto con las autoridades y profesores de Física correspondientes para solicitar la participación de los estudiantes de los Colegios y de la Universidad. En ambos casos el instrumento fue aplicado a todo el grupo clase, elegido al azar entre todos los existentes para cada nivel. Se administró en un aula con la colaboración del docente de la asignatura. 
Tabla 2: Contenido de las situaciones y representaciones simbólicas y pictóricas del instrumento.

\begin{tabular}{|l|c|c|c|c|}
\hline \multicolumn{2}{|c|}{ Contenido de las situaciones } & \multicolumn{2}{c|}{ Contenido de las representaciones simbólicas y pictóricas } \\
\hline Conceptos matemáticos & Conceptos físicos & Expresiones matemáticas, linguísticas y gráficas & Operaciones y Propiedades \\
\hline Escalar & $\begin{array}{c}\text { Masa, temperatura, } \\
\text { densidad, volumen, } \\
\text { energía, presión } \\
\text { carga,flujo } \\
\text { magnético } \\
\text { potencial }\end{array}$ & Número $\Re$ & Representación numérica $\Re$ & $\begin{array}{c}\text { Suma y resta } \\
\text { Producto }\end{array}$ \\
\hline Vector & $\begin{array}{c}\text { Fuerza, } \\
\text { desplazamiento, } \\
\text { velocidade, campos } \\
\text { eléctrico, magnético } \\
\text { gravitatorio }\end{array}$ & $\begin{array}{c}\text { Módulo } \Re \\
\text { Dirección y sentido }\end{array}$ & $\begin{array}{c}\text { Geométrica (flecha) } \\
\text { Análitica (componentes) }\end{array}$ & $\begin{array}{c}\text { Suma y resta } \\
\text { Producto escalar }\end{array}$ \\
\hline Función & $\begin{array}{c}\text { MRU } \\
\text { MRUA }\end{array}$ & $y=f(x)$ & $\begin{array}{c}\text { Gráfico } \\
\text { Ecuación }\end{array}$ & $\begin{array}{c}\text { Función lineal } \\
\text { Función cuadrática }\end{array}$ \\
\hline Campo escalar & $\begin{array}{c}\text { Campo de } \\
\text { temperaturas }\end{array}$ & $\phi=\phi(\vec{r}, t)$ & $\begin{array}{c}\text { Superfícies y curvas } \\
\text { de nivel }\end{array}$ & $\begin{array}{c}\text { Diferencial } \\
\text { Gradiente }\end{array}$ \\
\hline Campo vectorial & $\begin{array}{c}\text { Campo eléctrico, } \\
\text { Velocidad en un fluido } \\
\text { Otros }\end{array}$ & $\vec{A}(\vec{r}, t)$ & $\begin{array}{c}\text { Componentes } \\
\text { Diagramas de flechas } \\
\text { Líneas de campo }\end{array}$ & $\begin{array}{c}\text { Flujo } \\
\text { Circulación }\end{array}$ \\
\hline
\end{tabular}

Tabla 3. Síntesis análisis de consistencia interna del instrumento

\begin{tabular}{|l|l|l|l|l|}
\hline $\begin{array}{l}\text { Puntaje } \\
\text { total }\end{array}$ & $\begin{array}{l}\text { Media } \\
\text { puntaje total }\end{array}$ & $\begin{array}{l}\text { Desviación } \\
\text { Típica }\end{array}$ & $\begin{array}{l}\text { Número de } \\
\text { item }\end{array}$ & $\begin{array}{l}\text { Coeficiente } \\
\text { Alfa }\end{array}$ \\
\hline 80 & 37,36 & 11,61 & 5 & 0,71 \\
\hline
\end{tabular}

Tabla 4. Coeficientes de correlación de Pearson entre el puntaje total y el puntaje por item.

\begin{tabular}{|l|c|c|c|c|c|}
\hline Item & 1 & 2 & 3 & 4 & 5 \\
\hline Coeficiente de correlación de Pearson & 0,68 & 0,88 & 0,72 & 0,60 & 0,63 \\
\hline
\end{tabular}

\subsection{Análisis de datos}

Los datos obtenidos de las respuestas al instrumento fueron sometidos a un proceso de codificación, a partir del cual se establecieron cinco categorías de análisis que reflejaran de manera jerárquica los distintos elementos de los supuestos invariantes operatorios usados por los estudiantes en su interacción con los contenidos de la información de las situaciones y las representaciones simbólicas de los diferentes conceptos pertenecientes al campo conceptual del concepto de campo. Las categorías de análisis definidas son: Categoría 1: Clasificación; Categoría 2: Expresión escrita; Categoría 3: Representación; Categoría 4: Operación y Categoría 5: Resolución.

En la sección de Análisis de Resultados se describe cada una de estas categorías. La codificación de las respuestas se realizó en términos de tres niveles: correcto, incorrecto y no contesta, donde el nivel correcto describe en acuerdo con significados científicos de los conceptos, e incorrecto lo contrario. La justificación de este criterio adoptado es poder identificar la disponibilidad de conocimientos-en-acción científicamente aceptables en la estructura conceptual de los estudiantes, para aproximarse así, a la determinación de un nivel de conceptualización del concepto de campo. Una vez codificados los diferentes tipos de respuestas a las situaciones planteadas en el instrumento, los datos obtenidos se analizaron en términos de frecuencias. Por último, el desempeño obtenido por los participantes se asignó a un nivel de conceptualización del concepto de campo definido previamente a partir de las categorías de análisis señaladas anteriormente.

\section{Resultados}

Los resultados obtenidos se presentan en dos partes: La primera parte, se refiere a la descripción de las características de las respuestas científicamente correctas desplegados por los estudiantes sobre diferentes aspectos del campo conceptual del concepto de campo, determinadas a partir de las categorías de análisis, y la segunda parte, a la asignación de niveles de desarrollo conceptual del concepto de campo definidos a partir del desempeño en las categorías señaladas. 


\subsection{Características de las respuestas ci- entíficamente correctas}

De toda la información contenida en el instrumento aplicado, se presentan en esta sección los resultados referidos a los conocimientos científicamente correctos explicitados por los estudiantes. Para la determinación de estos resultados se definieron cinco categorías de análisis de las respues- tas de los estudiantes a las diversas situaciones planteadas en el instrumento (ver Tabla 5). El propósito de estas categorías es caracterizar, de acuerdo con la teoría de Vergnaud las relaciones de conocimiento entre la realidad de las situaciones y las respuestas (acción operatoria) de los estudiantes. A continuación se presentan los resultados del análisis del desempeño de los estudiantes en cada una estas categorías.

Tabla 5. Categorías de análisis de las respuestas de los estudiantes y contenidos del instrumento

\begin{tabular}{|c|c|c|c|}
\hline Clasificación & $\begin{array}{l}\text { Reconocimiento e identificación } \\
\text { de magnitudes físicas a las que se } \\
\text { aplican significados de los } \\
\text { conceptos de escalar, vector, } \\
\text { función, campo eléctrico en las } \\
\text { situaciones propuestas en el } \\
\text { instrumento }\end{array}$ & 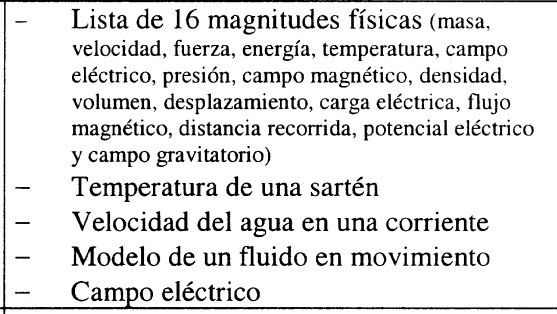 & $\begin{array}{c}6 \\
6 \\
7 \\
10 \\
\end{array}$ \\
\hline $\begin{array}{l}\text { Expresión } \\
\text { escrita }\end{array}$ & $\begin{array}{l}\text { Presencia de expresiones escritas } \\
\text { con predicados científicamente } \\
\text { correctos que definen atributos o } \\
\text { propiedades de los conceptos de } \\
\text { escalar, vector, función, campo } \\
\text { escalar y vectorial usadas para } \\
\text { explicar clasificaciones o } \\
\text { justificar respuestas a preguntas } \\
\text { contenidas en las situaciones. }\end{array}$ & $\begin{array}{ll}\text { Expresiones escritas: } \\
-\quad \text { Magnitudes clasificadas como vectores } \\
- & \text { Magnitudes clasificadas como escalares } \\
- & \text { Temperatura sartén como un campo } \\
& \text { escalar } \\
- & \text { Velocidad agua como campo vectorial } \\
- & \text { Situación cotidiana de un campo escalar } \\
- & \text { Situación cotidiana de un campo } \\
& \text { vectorial } \\
- & \text { Campo eléctrico como campo vectorial } \\
- & \text { Definición operacional de campo } \\
& \text { eléctrico }\end{array}$ & $\begin{array}{l}1 \\
1 \\
6 \\
6 \\
5 \\
5 \\
5 \\
10 \\
10 \\
\end{array}$ \\
\hline Representación & $\begin{array}{l}\text { Uso e identificación de } \\
\text { invariantes que se relacionen con } \\
\text { el conjunto de representaciones } \\
\text { simbólicas y pictóricas que } \\
\text { representen significados de los } \\
\text { conceptos de escalar, vector, } \\
\text { función, campo escalar, campo } \\
\text { vectorial y campo eléctrico. }\end{array}$ & $\begin{array}{ll}\text { Representaciones: } \\
- & \text { Vectores por flechas } \\
- & \text { Vectores por componentes } \\
- & \text { Función por ecuación } \\
- & \text { Función por gráfico } \\
- & \text { Fuerzas por flechas en un campo } \\
& \text { vectorial } \\
- & \text { Campo eléctrico por ecuaciones } \\
- & \text { Campo eléctrico por líneas de fuerza } \\
- & \text { Campo eléctrico por ecuaciones y líneas } \\
\end{array}$ & $\begin{array}{l}2 \\
2 \\
4 \\
4 \\
8 \\
\\
10 \\
10 \\
10\end{array}$ \\
\hline Operación & $\begin{array}{l}\text { Uso e identificación de } \\
\text { invariantes a partir de } \\
\text { procedimientos usados por los } \\
\text { estudiantes en las situaciones, } \\
\text { esto es, conocimiento y } \\
\text { aplicación de operaciones, } \\
\text { propiedades y representaciones } \\
\text { simbólicas ligadas a los } \\
\text { conceptos de escalar, vector, } \\
\text { función, campo escalar, campo } \\
\text { vectorial y campo eléctrico. }\end{array}$ & $\begin{array}{ll}\text { Operaciones: } \\
- & \text { Suma de vectores por flechas } \\
- & \text { Resta de vectores por flechas } \\
- & \text { Suma de vectores por componentes } \\
- & \text { Resta de vectores por componentes } \\
- & \text { Cálculo de vectores en un campo } \\
& \text { vectorial } \\
- & \text { Cálculo de flujo } \\
- & \text { Cálculo de circulación }\end{array}$ & $\begin{array}{c}2 \text { y } 3 \\
2 \\
2 \text { y } 3 \\
2 \\
8 \\
9 \\
9\end{array}$ \\
\hline Resolución & $\begin{array}{l}\text { Disponibilidad conceptual de } \\
\text { los estudiantes en términos de } \\
\text { propiedades, relaciones y } \\
\text { transformaciones científicamente } \\
\text { correctas de los conceptos de } \\
\text { escalar, vector, función y campo } \\
\text { en la resolución de un problema, } \\
\text { como manifestación del uso de } \\
\text { invariantes de las operaciones y } \\
\text { representaciones simbólicas de } \\
\text { los conceptos en acuerdo con las } \\
\text { demandas de un problema. }\end{array}$ & $\begin{array}{l}\text { Contenido y contexto de problemas: } \\
\text { - Álgebra vectorial, determinación del } \\
\text { - ángulo de las diagonales de una mesa. } \\
\text { Aplicación de la función lineal y } \\
\text { cuadrática en un problema de } \\
\text { cinemática. } \\
\text { - } \\
\text { Elección entre un modelo de sistema de } \\
\text { partículas o campo para un fluido. } \\
\text { - Descripción influencia de un campo de } \\
\text { fuerzas sobre una partícula por una } \\
\text { trayectoria. }\end{array}$ & $\begin{array}{l}7 \\
8\end{array}$ \\
\hline
\end{tabular}




\subsubsection{Clasificación}

Los resultados globales obtenidos en esta categoría en términos de frecuencia, se presentan en la Figura 1 y corresponden a presencia de clasificación correcta de las magnitudes físicas en acuerdo con la clase de conceptos de: escalar, vector, campo escalar, campo vectorial y campo eléctrico. La mayor frecuencia es la clasificación de magnitudes físicas con un 82,5\% de los estudiantes que clasifican correctamente como escalares o vectores las 16 magnitudes de la lista. En cambio, en una banda del 41,8 \% al 56,4\% de los estudiantes logra clasificar correctamente en las clases de campo escalar o vectorial las magnitudes físicas presentadas en el contexto de una situación cotidiana (temperatura de una sartén y velocidad de una corriente de agua), de un problema (modelo para un fluido en movimiento) y sobre carácter vectorial del campo eléctrico.

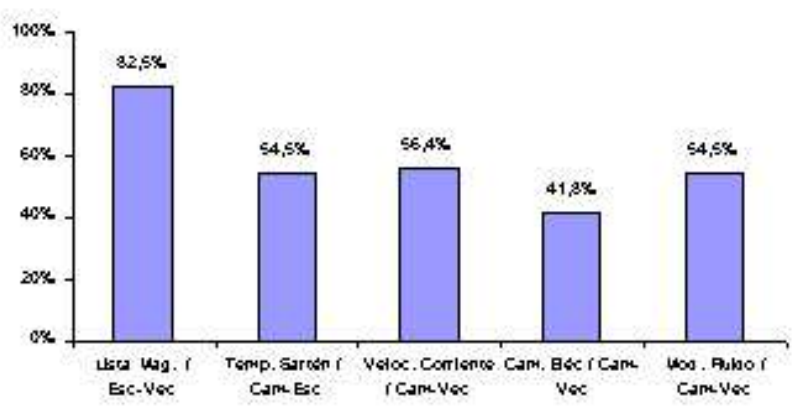

Figura 1. Porcentaje de estudiantes que clasifican de acuerdo con la clase de los conceptos de escalar, vector, campo escalar y vectorial y campo eléctrico.

De estos resultados parece inferirse dificultades de los estudiantes para transformar sus conocimientos-en-acción del concepto de campo a situaciones cotidianas y problemas, o bien, la ausencia de tal conocimiento. Por otra parte, cabe destacar, que un específico de los datos muestra que las magnitudes con menor frecuencia correcta de clasificación fueron Flujo magnético $(16,4 \%)$ y Potencial eléctrico $(54,5$ $\%)$ respectivamente. Estos valores están muy por debajo de las otras magnitudes y en forma preliminar podrían reflejar una falta de enseñanza de dichos conceptos, o una señal de ausencia de invariantes operatorios del concepto de campo por parte de los estudiantes, que se ponen de manifiesto cuando las magnitudes corresponden a conceptos físicos definidos a partir de operaciones más abstractas, propias de un campo vectorial.

\subsubsection{Expresión escrita}

Los resultados globales de esta categoría en términos de frecuencia se presentan en el Figura 2. Corresponden a la presencia en las respuestas de expresiones escritas que contienen predicados científicamente correctos que definen atributos o propiedades de los conceptos de escalar, vector, función, campo escalar, campo vectorial y campo eléctrico usados para explicar sus clasificaciones o justificar sus respuestas a preguntas contenidas en las diversas situaciones.

Los resultados de esta categoría muestran que el uso de expresiones escritas con explicitación de significados científicamente correctos de los conceptos usados para la justificación o explicación de las situaciones de clasificación es bajo, con frecuencias menores del $50 \%$. Estos valores están por debajo de los valores de frecuencia de clasificación correspondientes en la Figura 1.

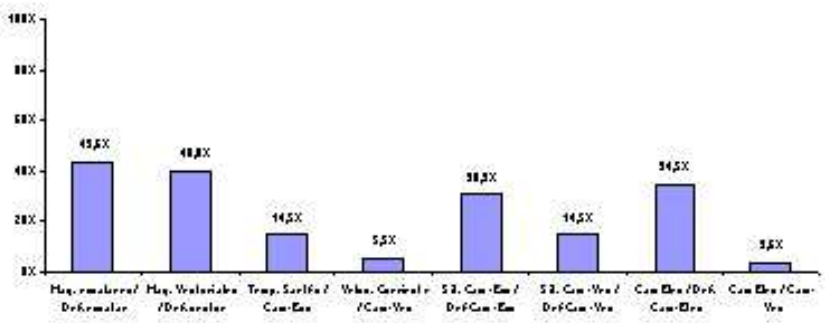

Figura 2. Porcentaje de estudiantes que utilizan expresiones escritas con significados científicamente correctos de los conceptos de escalar, vector, campo escalar y vectorial y campo eléctrico.

Si bien el 82,5\% clasifica correctamente como escalar o vector los elementos de la lista de 16 magnitudes, menos de la mitad de los estudiantes escriben expresiones científicamente correctas que explican la clasificación de estas magnitudes como escalares $(40,0 \%)$ y como vectoriales $(43,6 \%)$. Lo mismo ocurre con las frecuencias de las expresiones escritas usadas para justificar la clasificación de campo escalar de la temperatura de una sartén $(14,5 \%$,) de campo vectorial de la velocidad del agua en una corriente $(5,5 \%)$ y el campo eléctrico $(3,6 \%)$ respectivamente. Estos resultados podrían interpretarse como un indicador de dificultades de los estudiantes para utilizar sus conocimientosen-acción en situaciones que demandan un lenguaje de nivel conceptual mayor (Vergnaud,1998). En efecto, si se observan los resultados de las expresiones referidas al concepto de campo se constata que todas estas frecuencias están por debajo de las que corresponden a los conceptos de escalar y vector. También, llama la atención que los estudiantes puedan explicar en mayor proporción la clasificación del campo eléctrico como un campo vectorial $(34,5 \%)$ y que sólo un $3,6 \%$ escriba expresiones que justifiquen su comprensión del carácter de campo vectorial del campo eléctrico a partir de su definición operacional.

\subsubsection{Representación}

Los resultados globales para esta categoría se presentan en la Figura 3. Corresponden al uso de representaciones simbólicas (flechas, componentes, gráficos, ecuaciones) y pictóricas (líneas de fuerza) de conceptos matemáticos y físicos pertenecientes al campo conceptual del concepto de campo.

En las representaciones del concepto de vector se aprecia que la representación de vector por una flecha, que corresponde a una representación geométrica tipo imagen, es usada por el $100 \%$ de los estudiantes. Sin embargo, la representación de un vector por componentes, que corresponde a una representación analítica del tipo proposicional se reduce drásticamente a una frecuencia de $25,5 \%$. 


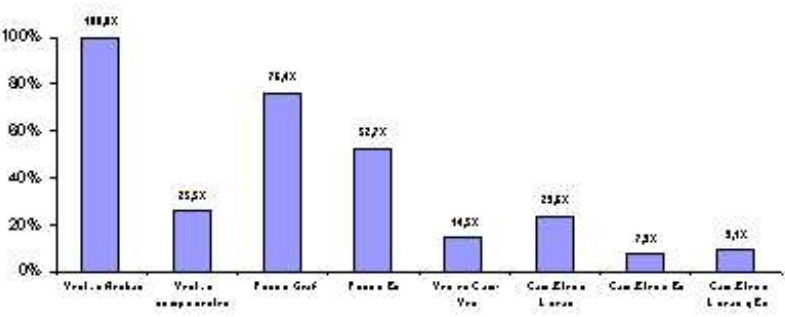

Figura 3. Porcentaje de estudiantes que utilizan representaciones simbólicas y pictóricas científicamente correctas de los conceptos de escalar, vector, campo escalar y vectorial y campo eléctrico.

Esta tendencia se mantiene para las representaciones de función, vectores fuerza en un campo vectorial y campo eléctrico, que corresponden a representaciones tipo imagen, es decir, las representaciones de gráficos, flechas y líneas de fuerza presentan frecuencias mayores que las representaciones proposicionales por ecuaciones. Por otra parte, los valores de frecuencia menores e iguales al 23,6 \% de las representaciones de campo eléctrico, muestran un bajo uso de representaciones simbólicas y pictóricas de este concepto, que se relaciona con los valores bajos de frecuencia de uso de expresiones escritas científicamente aceptables de la Categoría 2, que se refieren al carácter de campo vectorial del campo eléctrico. Este resultado puede ser interpretado desde la teoría de Vergnaud (1998) que describe dificultades de explicitación de los sujetos mediante el uso de representaciones simbólicas y lenguaje en situaciones que implican un mayor nivel de conceptualización.

\subsubsection{Operación}

Los resultados de esta categoría en términos de frecuencia se presentan en la Figura 4. Corresponden a la presencia en las respuestas científicamente correctas de uso de operaciones y propiedades de los conceptos de vector, función, campo escalar y campo vectorial.

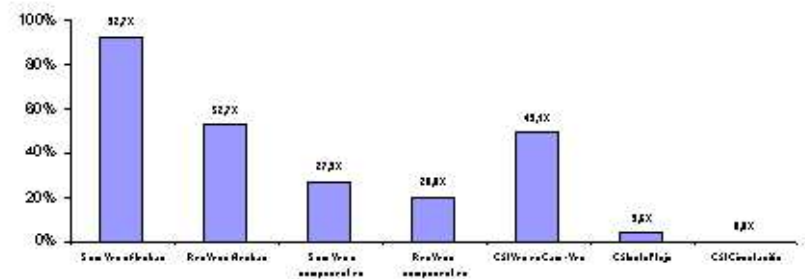

Figura 4. Porcentaje de estudiantes que utilizan operaciones científicamente correctas de los conceptos de vector campo vectorial.

Un análisis de los procedimientos usados por los estudiantes en operaciones muestra valores de desempeño correcto alto, para este nivel de instrucción, en operaciones del concepto de vector, con un $92,7 \%$ para la suma y $52,7 \%$ para la resta en la representación de vectores por flechas. Esta tendencia se mantiene para estas dos operaciones en la representación de componentes, pero los valores de frecuencia bajan a $27,3 \%$ para la suma y $20,0 \%$ para la resta. Si se comparan estos resultados con el desempeño en la categoría 3 , de representación del concepto de vector, se observa un comportamiento similar, que parece evidenciar dificultades de los estudiantes para alcanzar a apropiarse de invariantes que reflejen una conceptualización más abstracta, como el uso de representaciones analíticas de las operaciones y propiedades del álgebra vectorial. El desempeño en las operaciones asociadas al concepto de campo vectorial es bajo; el mejor desempeño es un 49,1\% que corresponde, a estudiantes que calcularon correctamente en puntos del espacio, los vectores de un campo de fuerzas presentado mediante una expresión analítica. No obstante, si se observa los resultados correspondientes en la categoría 3, se comprueba que la representación correcta de estos mismos vectores es un $14,5 \%$. Este resultado evidencia dificultades para ubicar y representar los vectores en puntos del espacio del campo de fuerzas, poniendo de manifiesto incomprensión del concepto de campo como una función, que asocia valores de fuerza a cada punto del espacio.

Por otra parte, el bajo desempeño de un 3,6\% en el cálculo del flujo y nulo de la circulación, podría explicarse como un desconocimiento de estas operaciones y conceptos en estos niveles educativos, o bien podría estar mostrando, en acuerdo con la teoría de Vergnaud (1998), la ausencia de invariantes más complejos que demandan niveles de conceptualización más altos. Este bajo desempeño en el cálculo del flujo se relaciona en un mismo sentido con el bajo desempeño mencionado en la categoría 1 de clasificación de estos conceptos como magnitudes escalares.

\subsubsection{Resolución}

Los resultados de esta categoría se presentan en la Figura 5 y corresponden a la disponibilidad conceptual de los estudiantes, en términos de propiedades, relaciones y transformaciones científicamente correctas de los conceptos de vector, función y campo vectorial en la resolución de un problema.

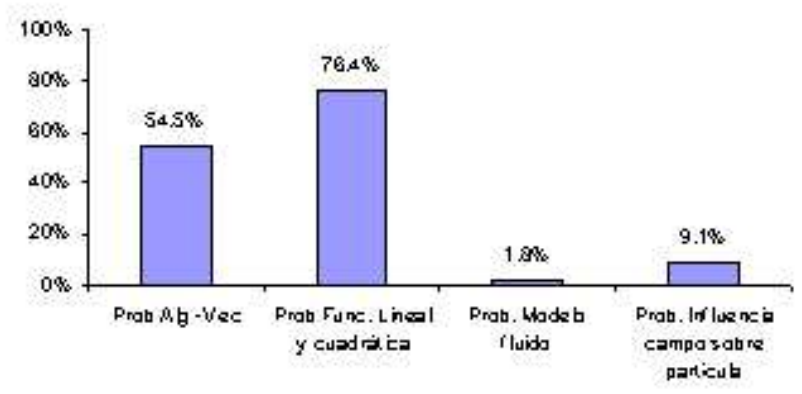

Figura 5. Porcentaje de estudiantes con disponibilidad conceptual en términos de propiedades, relaciones y transformaciones científicamente correctas de los conceptos de vector, función y campo vectorial en la resolución de un problema.

Los mayores valores de frecuencia de disponibilidad conceptual se observan en la resolución de problemas de aplicación de los conceptos de vector $(54,5 \%)$ y función $(76,4 \%)$ respectivamente. En cambio, los estudiantes muestran una disponibilidad conceptual muy baja para enfrentar problemas que demandan el uso y aplicación del concepto de campo científicamente aceptable, con valores de un 1,8 $\%$ para el problema de explicar y justificar la elección de un modelo de fluido en movimiento y de 9,1\% para el problema 
de describir y explicar la influencia de un campo de fuerzas sobre una partícula. Estos resultados muestran una baja aprehensión del campo conceptual del concepto que podría tener como origen, de acuerdo con la teoría de Vergnaud, el hecho que los estudiantes no otorgan sentido a las situaciones y problemas desde invariantes que dan significado al concepto de campo.

En resumen, los resultados observados en todas las categorías anteriores respecto del concepto de campo, además del bajo uso de invariantes que operan sobre los objetos físicos y matemáticos y sus representaciones simbólicas para enfrentar las demandas de problemas de aplicación, pa- recería reflejar bajos niveles de conceptualización de los estudiantes del concepto de campo.

\subsection{Niveles de conceptualización del concepto de campo}

Con el propósito describir grados de dominio del campo conceptual del concepto de campo, se definieron cinco niveles de conceptualización a partir del desempeño mostrado por los estudiantes en las diferentes categorías. La descripción de cada nivel se presenta en la Tabla 6.

Tabla 6. Descripción de niveles de conceptualización del concepto de campo.

\begin{tabular}{|c|c|c|}
\hline Nivel & Descripción & Ejemplo \\
\hline $\mathrm{N}_{0}$ & $\begin{array}{l}\text { Ausencia de invariantes operatorios adecuados de comprensión del con- } \\
\text { cepto de campo: El sujeto no contesta o escribe respuestas irrelevantes, } \\
\text { o no utiliza la palabra campo en sus explicaciones, o confunde el término } \\
\text { campo estableciendo vínculos con un dominio o campo disciplinario. }\end{array}$ & $\begin{array}{l}\text { El dinero en un banco es un campo por- } \\
\text { que pertenece al "...campo de la eco- } \\
\text { nomía..." }\end{array}$ \\
\hline $\mathrm{N}_{1}$ & $\begin{array}{l}\text { Reconocimiento de un campo sin explicación de significados ci- } \\
\text { entíficamente aceptados del concepto: En este nivel, el sujeto aún no ma- } \\
\text { nifiesta una conceptualización del concepto de campo, sino sólo clasifica } \\
\text { magnitudes, que si bien se describen mediante el concepto de campo, aún } \\
\text { no explica o usa operaciones y representaciones simbólicas que reflejen } \\
\text { una comprensión de significados científicamente aceptables del concepto. }\end{array}$ & $\begin{array}{l}\text { La velocidad de una corriente "es un } \\
\text { campo vectorial...". Reconoce el carácter } \\
\text { vectorial sin explicar significados del } \\
\text { concepto de campo. }\end{array}$ \\
\hline $\mathrm{N}_{2}$ & $\begin{array}{l}\text { Reconocimiento de un campo y explicación parcial de significados ci- } \\
\text { entíficamente aceptados del concepto: El sujeto sólo reconoce situa- } \\
\text { ciones y clasifica magnitudes físicas a las que aplica el concepto de } \\
\text { campo con explicaciones que sólo se refieren a aspectos parciales del } \\
\text { concepto. Usa en forma limitada algunas operaciones y representaciones } \\
\text { simbólicas, pero no alcanza a vincularlas como una forma de represen- } \\
\text { tación u operación ligada al concepto de campo. No se alcanza a inferir } \\
\text { una aplicación de conocimientos-en-acción del concepto en la resolución } \\
\text { de un problema }\end{array}$ & $\begin{array}{l}\text { La temperatura en un sartén es un campo } \\
\text { escalar, porque "irradia desde el centro } \\
\text { hasta hacerse homogénea..." } \\
\text { Calcula correctamente los vectores de un } \\
\text { campo de fuerzas para diversos puntos de } \\
\text { un plano, pero los dibuja todos juntos en } \\
\text { un mismo punto. }\end{array}$ \\
\hline $\mathrm{N}_{3}$ & $\begin{array}{l}\text { Transición entre un reconocimiento y significación parcial del concepto } \\
\text { con aplicación del concepto de campo a situaciones y problemas: El su- } \\
\text { jeto reconoce situaciones y clasifica magnitudes físicas a las que aplica } \\
\text { el concepto de campo. En sus explicaciones se refleja organización y } \\
\text { comprensión de los significados de los conceptos, operaciones y repre- } \\
\text { sentaciones simbólicas del concepto de campo, pero aún no logra conec- } \\
\text { tarlas completamente. Aplica el concepto de campo en la resolución de } \\
\text { un problema }\end{array}$ & $\begin{array}{l}\text { Velocidad del agua en una corriente "es } \\
\text { un campo vectorial porque depende de } \\
\text { sus coordenadas y de su posición...”. El } \\
\text { mismo sujeto en otra situación afirma } \\
\text { “...un fluido es un medio homogéneo y } \\
\text { continuo en que sus propiedades son con- } \\
\text { tinuas en todo su ser...” para explicar que } \\
\text { un modelo de sistema de partículas des- } \\
\text { cribe un fluido en movimiento. }\end{array}$ \\
\hline $\mathrm{N}_{4}$ & $\begin{array}{l}\text { Aprehensión del concepto de campo para el nivel de instrucción: El su- } \\
\text { jeto manifiesta comprensión y explicitación de los significados del con- } \\
\text { cepto, para describir el comportamiento de una magnitud física definida } \\
\text { en cada punto de una región del espacio y del tiempo, como también hace } \\
\text { un uso apropiado de las operaciones, representaciones simbólicas y pro- } \\
\text { piedades del concepto de campo en situaciones y problemas. }\end{array}$ & \\
\hline
\end{tabular}


Tabla 7. Escala de desempeño para la determinación de niveles de conceptualización.

\begin{tabular}{|l|l|l|l|l|l|}
\hline $\begin{array}{l}\text { Rango } \\
\text { desempeño } \\
\text { Nivel }\end{array}$ & $\begin{array}{l}\text { Clasificación } \\
(4)\end{array}$ & $\begin{array}{l}\text { Expresión } \\
\text { escrita } \\
(6)\end{array}$ & $\begin{array}{l}\text { Representación } \\
(4)\end{array}$ & $\begin{array}{l}\text { Operación } \\
(3)\end{array}$ & $\begin{array}{l}\text { Resolución } \\
(2)\end{array}$ \\
\hline $\mathbf{0}$ & & & & & \\
\hline $\mathbf{1}$ & $1-2$ & 1 & & & \\
\hline $\mathbf{2}$ & $1-2$ & 1 & 1 & 1 & 1 \\
\hline $\mathbf{3}$ & $1-2$ & $1-4$ & $1-2$ & 1 & 1 \\
\hline $\mathbf{4}$ & $3-4$ & $5-6$ & $1-2$ & $1-2$ & 1 \\
\hline
\end{tabular}

La asignación de los estudiantes en cada nivel de conceptualización, se realizó a partir de su desempeño individual, en las respuestas referidas exclusivamente al concepto de campo en cada una de las categorías. Con este propósito se definió una escala de desempeño aceptable por rangos, para las respuestas científicamente correctas, por categoría en cada uno de los niveles de conceptualización. Esta escala se presenta en la Tabla 7, y los valores que aparecen en paréntesis corresponden al número de respuestas posibles en cada categoría.

La distribución de los estudiantes de la muestra en los cinco niveles de conceptualización del concepto de campo se presenta en la Figura 6.

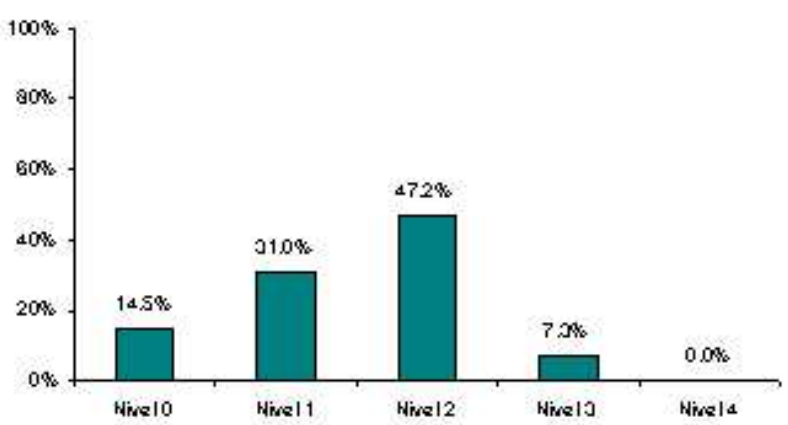

Figura 6. Porcentaje de estudiantes ubicados en los niveles de conceptualización del concepto de campo.

El 47,2 \% de los estudiantes se ubica en el Nivel 2 de conceptualización, que corresponde sólo a un reconocimiento y explicación parcial de significados del concepto de campo. Se observa que el 45,5\% de los estudiantes se sitúa en los dos niveles más bajos de conceptualización Nivel 0 $(14,5 \%)$ y Nivel $1(31,0 \%)$ respectivamente. De acuerdo a la teoría de Vergnaud (1998) estos resultados estarían mostrando ausencia de esquemas que contengan invariantes para enfrentar situaciones que demanden explicaciones y predicciones científicamente aceptables de aplicación del concepto campo. Además, sólo el 7,3\% de los estudiantes se ubica en el Nivel 3 de transición y ningún estudiante en el Nivel 4 de aprehensión del concepto de campo.

Los niveles bajos de conceptualización de la mayoría de los estudiantes pueden interpretarse mejor, si se analizan los desempeños en las categorías usadas para determinar los niveles de conceptualización. Los resultados de este análisis se presentan en la Figura 7.

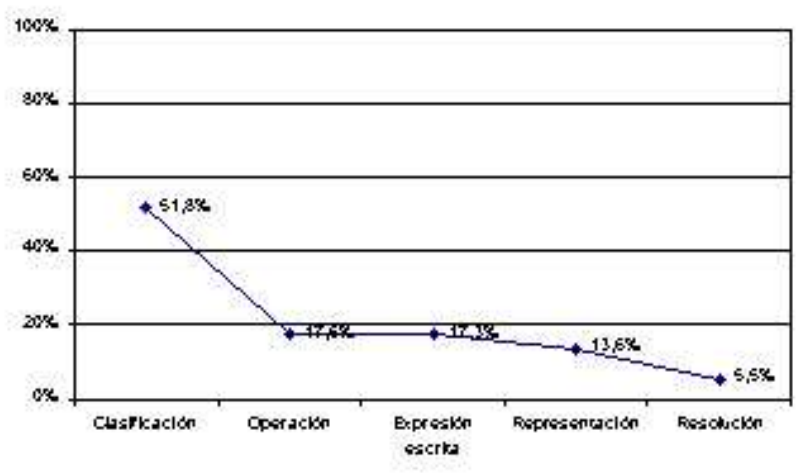

Figura 7. Desempeño de los estudiantes por categorías en la determinación de niveles de conceptualización.

Se aprecia que la mayoría de los estudiantes tuvo desempeños menores al $20 \%$ en todas las categorías que exigen un mayor uso de significados científicamente correctos del concepto de campo. Si se observan los valores en las categorías de operación, expresión escrita, representación y resolución, se constata una tendencia descendente monótona con valores más altos en las categorías de menor exigencia conceptual.

\section{Conclusión y discusión}

Se observa que la mayoría de los estudiantes de la muestra presentan una ausencia notoria de invariantes operatorios para enfrentar las situaciones y problemas propuestos en esta investigación. El 92,7\% de los estudiantes se ubica en los tres niveles más bajos de conceptualización (Niveles 1, 2 y 3) del concepto de campo definidos en este estudio, donde el Nivel 3 corresponde tan sólo a alcanzar un reconocimiento y explicación parcial de significados del concepto de campo. Esta clasificación de bajo nivel de conceptualización es coherente con los bajos desempeños en las categorías de operación $(17,6 \%)$, expresión escrita $(17,3 \%)$, representación $(13,6 \%)$ y resolución $(5,5 \%)$ y está en acuerdo con la teoría de que afirma que un bajo nivel de conceptualización se caracteriza por bajos niveles de explicitación de invariantes y sus representaciones, con predominio de los aspectos procedimentales de las operaciones por sobre el uso de predicados que expresen una mayor riqueza conceptual (Vergnaud, 1998).

En efecto, si se analizan los desempeños de la categoría de operación (Fig. 4), el predominio de los aspectos proce- 
dimentales se confirma, ya que en las operaciones ligadas al concepto de campo, la mayor frecuencia de desempeño es un $49,1 \%$, que corresponde al cálculo de vectores de fuerza de un campo vectorial a partir de su expresión analítica, que exige evaluar y representar una función en forma correcta en puntos del espacio pertenecientes a su dominio. Sin embargo, en otras operaciones más complejas, que describen propiedades de invariantes físicos $I(F F)$ propios del concepto de campo y claves para la descripción de fenómenos físicos, mencionados en la descripción del campo conceptual del concepto de campo construido por la física (ver p. 8 y 9), tales como el flujo y la circulación, el desempeño de los estudiantes es muy bajo, con valores de $3,6 \%$ y cero respectivamente. Si bien, una posible explicación de estos desempeños tan bajos podría estar en un desconocimiento de estas operaciones y conceptos en los estudiantes de Bachillerato, que representan a un $87 \%$ de los estudiantes de la muestra, estos valores aún estarían por debajo de los valores esperados, que deberían estar en torno del $13 \%$ que corresponde a la proporción de estudiantes de nivel universitario. En consecuencia, los niveles bajos de conceptualización mostrado por el $92,7 \%$ de los estudiantes se relacionan, más bien, con niveles bajos de explicitación de invariantes físicos del concepto de campo, con predominio de aspectos procedimentales de las operaciones asociadas a invariantes de objetos matemáticos $I(O M)$ del campo conceptual del concepto de campo, como lo corroboran los desempeños de las operaciones suma y resta de vectores, mostrados también en la Fig. 4, cuyos valores están por encima de los desempeños asociados a las operaciones ligadas a invariantes propios del concepto de campo.

Por otra parte, el hecho que el 7,3\% de los estudiantes, se ubique en el Nivel 3 de conceptualización (de transición) y ninguno en el Nivel 4 (de aprehensión) del concepto de campo, también es coherente con los resultados de desempeño en la categoría de resolución (Fig. 5), en la cual se observa que la disponibilidad conceptual es escasa $(1,8$ $\%$ en el problema de elegir y justificar un modelo de campo para un fluido en movimiento, y 9,1\% en otro, de describir la acción de un campo sobre una partícula), en términos de propiedades, relaciones y transformaciones científicamente aceptables del concepto de campo.

Desde la teoría de campos conceptuales, estos resultados indican que la mayoría de los estudiantes no dan sentido a los problemas desde invariantes que otorgan significado al concepto de campo, es decir, se evidencia ausencia de invariantes físicos y matemáticos del concepto de campo construido por la física, lo cual concuerda con resultados de otras investigaciones (Furió y Guisasola, 2001; Guisasola, 2001). Sin embargo, es importante destacar, que un 54,5 $\%$ de los estudiantes presenta una disponibilidad conceptual científicamente aceptable para enfrentar un problema de geometría, de aplicación de álgebra vectorial, y un 76,4 \% en otro de cinemática de aplicación del concepto de función; esto indica que los estudiantes otorgan sentido a problemas desde invariantes de conceptos pertenecientes tales como vector y función, pero sin alcanzar aún a acomodar estos invariantes en esquemas más generales que pertenezcan a representaciones del concepto de campo. Una situación si- milar se observa, al comparar el desempeño en el cálculo de vectores fuerza de un campo vectorial $(49,1 \%)$ en la categoría de operación (Fig. 4), con el desempeño menor de un 14,5\% en la categoría de representación (Fig.3), para la representación de vectores fuerza en el espacio de su dominio; esto significa que de aquellos estudiantes que poseen invariantes apropiados para enfrentar situaciones que incluyen representaciones del concepto de función, una minoría ha logrado acomodarlos a esquemas de representación del concepto de campo.

Esta falta de generalidad de los esquemas, permite interpretar el mayor desempeño en todas las categorías respecto a los conceptos de escalar, vector y función, en comparación al desempeño menor en las respuestas relacionadas con el concepto de campo. En efecto, en Clasificación, las clasificaciones de escalar y vector tienen un desempeño del 83,6 \% y las clasificaciones relacionadas con el concepto de campo muestran valores menores e iguales al 54,6 \%. Lo mismo ocurre en expresiones para explicar clasificaciones, que usan significados de los conceptos de escalar y vector, (expresión escrita 43,0 \% y las del concepto de campo entre un 34,5\% y un 3,6\%). Igualmente, en la categoría de representación, las representaciones de los conceptos de vector y función se ubican en un rango del $100 \%$ al 25,5\%, y las de campo se distribuyen en un rango menor del $14,5 \%$ y $7,3 \%$; y en la categoría de operación, los desempeños en concepto de vector se distribuyen entre un $92,7 \%$ y un $20,0 \%$, en cambio, en el concepto de función en un rango de 49,1\% y 3,6 $\%$. En consecuencia, esta superioridad de desempeño en todas las respuestas afines a los conceptos de escalar, vector y función por sobre el desempeño en las respuestas asociadas al concepto de campo en todas las categorías, muestra que pocos estudiantes han logrado transponer o acomodar las representaciones de estos conceptos a representaciones del concepto de campo. Es decir, pocos estudiantes en su interacción con las situaciones y problemas manifiestan una apropiación parcial del campo conceptual del concepto de campo, lo cual podría explicar que un 7,3\% de los estudiantes se ubique en el Nivel 3 de transición, y ninguno el Nivel 4, de aprehensión del concepto de campo.

Otro aspecto importante de destacar de las respuestas de los estudiantes en las situaciones, es el mejor desempeño en el uso de representaciones simbólicas geométricas y pictóricas, tipo imagen por sobre el uso de representaciones analíticas de tipo proposicional. En la categoría de representación (Fig. 3), los desempeños en la representación de vectores por flechas y función por gráfico, tienen valores entre un $100 \%$ y $76,4 \%$, en cambio, las representaciones de vector por componente y función por ecuación presen$\tan$ un desempeño menor entre $52,7 \%$ y un $25,5 \%$. Las representaciones de campo eléctrico son similares, el desempeño de representación del campo eléctrico por líneas de fuerzas es $23,6 \%$ y la representación mediante ecuaciones es 7,3\%. Esta tendencia de predominio de uso de representaciones geométricas por sobre las analíticas, se mantiene en la categoría de operación, con desempeños para la suma y resta de vectores mediante flechas de $92,7 \%$ y $52,7 \%$ respectivamente, en cambio, para las mismas operaciones mediante componentes el desempeño es de $27,3 \%$ 
y $20 \%$. Por otra parte, respecto al uso de representaciones simbólicas lingüísticas, se observa en la categoría expresión escrita (Fig. 2), que el desempeño en las expresiones escritas utilizadas para justificar clasificaciones mediante los conceptos de escalar $(43,6 \%)$ y vector $(40 \%)$ está por encima de los desempeños en expresiones escritas para justificar clasificaciones y explicar situaciones usando el concepto de campo. Este hecho, podría estar mostrando una disponibilidad y uso limitados de instrumentos semióticos de representación simbólica de los estudiantes (Vergnaud, 1998) para dar significado a sus acciones en las situaciones, lo que conlleva, a dificultades para asimilar conceptos de mayor complejidad y abstracción como es el concepto de campo.

Por todo lo planteado, se puede concluir que los resultados obtenidos verifican algunas de las estructuras de representaciones simbólicas y significados, que dan forma a los invariantes operatorios que utilizan los estudiantes en situaciones y problemas que involucran una conceptualización científicamente aceptable del concepto de campo, y confirman lo sugerido por Vergnaud $(1981 ; 1998)$, que los conceptos no sólo deben ser definidos por su estructura, sino que requiere considerar, las propiedades, las situaciones en las cuales los conceptos son usados y los sistemas de representación simbólica (signos) que los estudiantes usan para pensar y escribir acerca de un concepto, o sea, la conceptualización está regulada por la interacción entre la información contenida en las situaciones y la estructura conceptual del estudiante.

Las implicaciones para futuras investigaciones apuntan a la consolidación de una metodología, para mejorar la caracterización de las representaciones o conocimientos-enacción, largamente implícitos, de los estudiantes del campo conceptual del concepto de campo, a partir de un análisis y revisión crítica del instrumento utilizado; y el diseño y aplicación de otros instrumentos como, entrevistas sobre situaciones y ejemplos; entrevistas sobre tareas para demostrar, observar y explicar situaciones; cuestionarios de respuesta cerrada y abiertas. Lo anterior permitiría abordar estudios, como investigar diferencias entre invariantes operatorios del concepto de campo construidos por grupos de estudiantes con distintos nivel de instrucción, o estudiar si los invariantes operatorios que se detectan dificultan la comprensión del concepto de campo, entonces a partir de este conocimiento, determinar criterios para evaluar propuestas didácticas, y a su vez, elaborar nuevas propuestas que faciliten un aprendizaje significativo del concepto de campo.

\section{Bibliografía}

Berkson, W., (1981). Las teorías de los campos de fuerza desde Faraday hasta Einstein. Madrid: Alianza.

Borges, A. T., Gilbert, J. K., (1998). Models of magnetism. International Journal of Science Education, 20(3); 361-378. Cronbach, L. J., (1967). Coefficient alpha and internal structure of tests.En W.A. Mehrens y R.L. Lebel (Org.) Principles of educational and psychological measurement. Chicago: Rand McNally.

Delval, J., (1997). Tesis sobre el constructivismo. En Rodrigo, M. J. y Arnay, J. (Eds) La construcción del conocimiento escolar, Barcelona: Paidós.
Di Sessa, A., Sherin, B. L, (1998). What changes in conceptual change?. International Journal of Science Education, 20 (10); 1155-1191.

Driver, R., Guesne, E. y Tiberghien. A., (1985). Children's ideas in science. Milton Keynes: Open University Press. Trad. Cast de P. Manzano: Ideas científicas en la infancia y en la adolescencia, Madrid: Morata /MEC, 1989.

Einstein, A., (1995). Sobre la teoría de la relatividad especial y general, Madrid: Alianza.

Feynman, R., (1985). Electrodinámica cuántica, Madrid: Alianza.

Furió, C., Guisasola, J., (1998a). Dificultades de aprendizaje de los conceptos de carga y de campo eléctrico en estudiantes de bachillerato y universidad. Enseñanza de las Ciencias, 16 (1); 131-146.

Furió, C., Guisasola, J., (1998b). Difficulties in learning the concept of electric field, Science Education, 82, 511-526.

Furió, C., Guisasola, J., (2001). La enseñanza del concepto de campo eléctrico basado en un modelo de aprendizaje como investigación orientada. Enseñanza de las Ciencias, 19 (2); 319-334.

Furió, C., (2001). La enseñanza-aprendizaje de las ciencias como investigación: Un modelo emergente. En Guisasola, J. Y Pérez de Eulate González (Eds). Investigaciones en didáctica de las ciencias experimentales basadas en el modelo enseñanza-aprendizaje como investigación orientada, Bilbao: Universidad del País Vasco.

Galili, I., (1995). Mechanics background influences student' conceptions in electromagnetism. International Journal of Science Education, 17 (3); 371-387.

Gil, D., Carrascosa, J., (1985). Science learning as a conceptual and methodological change. European Journal of Science Education, 7 (3); 231-236.

Gil, D., (1993). Contribución de la Historia y Filosofía de las ciencias al desarrollo de un método de enseñanza/aprendizaje como investigación. Enseñanza de las Ciencias, 11 (2); 197-212.

Greca, I., Moreira, M. A., (1997). The kinds of mental representation -models, propositions and images- used by college physics students regarding the concept of field. International Journal of Science Education, 19 (6); 711-724.

Greca, I., Moreira, M. A., (1998). Modelos mentales y aprendizaje de física en electricidad y magnetismo. Enseñanza de las Ciencias, 6 (2); 289-303.

Greca, I., Moreira, M. A., (2000). Mental models, conceptual models, and modelling. International Journal of Science Education, 22 (1); 1-11.

Greca, I., Moreira, M. A., (2002). Além da detecçao de modelos mentais dos estudantes. Uma proposta representacional integradora, Investigaçoes em Ensino de Ciências, 7 (1) $25-53$.

Guisasola, J., (2001). Análisis crítico de la enseñanza de la electrostática en el Bachillerato y propuesta alternativa de orientación constructivista. En Guisasola, J. Y Pérez de Eulate González (Eds) Investigaciones en didáctica de las ciencias experimentales basadas en el modelo enseñanzaaprendizaje como investigación orientada, Bilbao: Universidad del País Vasco. 
Harman, P. M., (1990). Energía, fuerza y materia. El desarrollo conceptual de la física del siglo XIX, Madrid: Alianza Universidad.

Johnson-Laird, P.N., (1983). Mental Models. Cambridge: MA, Harvard University Press.

Lakoff, G., (1999). Cognitive models and prototype theory. In E. Margolis, E y S. Laurence (Eds) Concepts - Core readings, Cambridge, MA: MIT Press.

Laurence, S., Margolis, E., (1999). Concepts and cognitive science. In E. Margolis, y S. Laurence (Eds) Conceps-Core readings, Cambridge, MA: MIT Press.

Landau, L. D., Lifshitz, E. M., (1992). Teoría clásica de los campos, Barcelona: Reverté.

Llancaqueo, A., Caballero, C., Moreira. (2003). El concepto de campo en el aprendizaje de la física $\mathrm{y}$ en la investigación en educación en ciencias. Revista Electrónica de Enseñanza de las Ciencias,2, 3 , Artículo 2. En http://www.apastyle.org/elecref.html.

Marín, N., (1999). Delimitando el campo de aplicación del cambio conceptual. Enseñanza de las Ciencias, 17 (1); 8092.

Martín, J., Solbes, J., (2001). Diseño y evaluación de una propuesta para la enseñanza del concepto de campo en física. Enseñanza de las Ciencias, 19 (3); 393-403.

Medin, D.1., (1998). Concepts and conceptual structure. In P. Thagard (Ed) Mind reading, Cambridge, MA: MIT Press. Moreira, M. A., (1994). Cambio conceptual: crítica a modelos y una propuesta a 1 luz de la teoría del aprendizaje significativo. In : Science \& Mathematics Education for the $21^{\text {st }}$. Century: Towards innovatory approaches. Concepción, Chile. Actas, 81-92.

Moreira, M. A., (1998). La investigación en educación en ciencias y la formación permanente del profesor de ciencias, Conferencia presentada en Congreso Iberoamericano de Educación en Ciencias Experimentales, La Serena, Chile.

Moreira, M. A., 2000. Aprendizaje significativo: teoría y práctica, Madrid: Visor.

Moreira, M. A., (2002). A Teoría dos campos conceituais de Vergnaud, o ensino de ciências e a pesquisa nesta área, Investigaçoes em Ensino de Ciências, 7 (1); 1-24.

Palmer, D., (2002). Students' alternative conceptions and scientifically acceptable conceptions about gravity. International Journal of Science Education, 23 (7); 691-706.

Pauli, W., (1996). Escritos sobre física y filosofía, Madrid: Debate.

Pozo, J. I., Pérez Echeverría, M. P.; Sanz, A. y Limón, M., (1992). Las ideas de los alumnos sobre la ciencia como teorías implícitas. Infancia y Aprendizaje, 57 ; 3-32.

Pozo, J. L., (1996); La psicología cognitiva y la educación científica, Investigações em Ensino de Ciências, 1 (2): 1-25. Pozo, J. I., Gómez Crepo, M. A., (1998). Aprender y enseñar ciencia. Del conocimiento cotidiano al conocimiento científico, Madrid: Morata.

Pozo, J. I., (1999). Más allá del cambio conceptual: El aprendizaje de la ciencia como cambio representacional. Enseñanza de las Ciencias, 17 (3); 513- 520.

Pozo, J. I., Rodrigo, M. J., (2001). Del cambio de contenido al cambio representacional en el conocimiento conceptual,
Infancia y Aprendizaje, 24 (4); 407-423.

Pozo, J. L., (2002); La adquisición del conocimiento científico como un proceso de cambio representacional, Investigações em Ensino de Ciências, 7 (3): 1-30.

Rodríguez, M., (1999). Conocimiento previo y cambio conceptual, Buenos Aires : Aique.

Silveira, F. L. da., (1981). Fidedignidade das medidas e diferenças entre grupos em psicologia e educaçao. Ciência e Cultura, 33 (5); 704-707.

Silveira, F. L., (1993). Validaçao de testes de papel e lápis. En M. Moreira y F. L. Silveira (Ed.), Instrumentos de pesquisa em ensino e aprendizagem. Porto Alegre:Edipucrs.

Slater, J., Frank, N., (1947). Electromagnetism, New York: Mc Graw-Hill Company, Inc..

Vergnaud, G., Halwachs, F., Rouchier, A., (1981). Estructura de la materia enseñada, historia de las ciencias y desarrollo conceptual del alumno. En Coll, C. (Ed) Psicología genética y educación, Barcelona: Oikos-tau.

Vergnaud, G., (1990). La théorie des champs conceptuels. Recherches en Didatique des Mathématiques, 10 (23); 133170

Vergnaud, G., (1993). Teoria dos campos conceituais. In Nasser, L. (Ed) Anais do $1^{o}$ Seminario Internacional de Educaçao Matemática do Rio de Janeiro; 1-26.

Vergnaud, G., (1996). Algunas ideas fundamentales de Piaget en torno a la didáctica. Perspectivas, 26 (10) ; 195-207.

Vergnaud, G., (1998). A comprehensive theory of representation for mathematics education. Journal of Mathematical Behaviour, 17 (2); 167-181.

Viennot, L., Rainson, S., (1992). Students' reasoning about the superposition of electric field. International Journal of Science Education, 14 (4); 475-487.

Viennot, L., Rainson, S., (1999). Design and evaluation of research-based teaching sequence: the superposition of electric field. International Journal of Science Education, 21 (1); 1-16.

Viennot, L., (2002). Razonar en física. La contribución del sentido común, Madrid: Antonio Machado.

\section{Anexo 1}

\section{Instrumento}

1. De las siguientes magnitudes físicas:

Masa; Velocidad; Fuerza; Energía; Temperatura; Campo eléctrico; Presión; Campo magnético; Densidad; Volumen; Desplazamiento; Carga eléctrica; Flujo magnético; Distancia recorrida; Potencial eléctrico; Campo gravitatorio.

Clasifica en dos grupos, aquellas que consideres magnitudes "escalares" y aquellas que consideres magnitudes "vectoriales” y explica ¿Por qué? 


\begin{tabular}{|l|l|l|l|}
\hline $\begin{array}{l}\text { Magnitudes } \\
\text { escalares }\end{array}$ & Explicación & $\begin{array}{l}\text { Magnitudes } \\
\text { vectoriales }\end{array}$ & Explicación \\
\hline & & & \\
\hline & & & \\
\hline & & & \\
\hline & & & \\
\hline & & & \\
\hline & & & \\
\hline & & & \\
\hline & & & \\
\hline & & & \\
\hline
\end{tabular}

2. Los lados de la superficie de una mesa rectangular como la que se muestra en la figura miden 40 x $30 \mathrm{~cm}$ y los vértices de cada esquina se designan por las letras A, B , C y D.

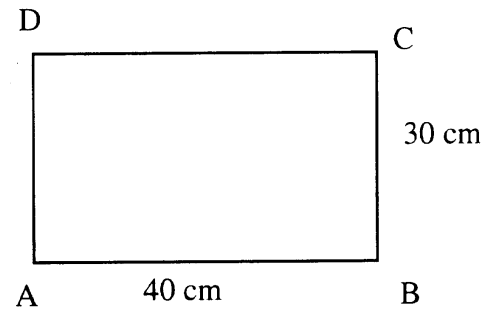

- Si $A \vec{B}$ y $A \vec{D}$ son vectores que unen los vértices $\mathrm{AB}$ y $\mathrm{AD}$ respectivamente, dibuja en la figura los vectores: $A \vec{B} ; A \vec{D} ; A \vec{B}+A \vec{D}$ y $A \vec{D}-A \vec{B}$

- Si se define un sistema de referencia con origen en el vértice A como se muestra en la siguiente figura

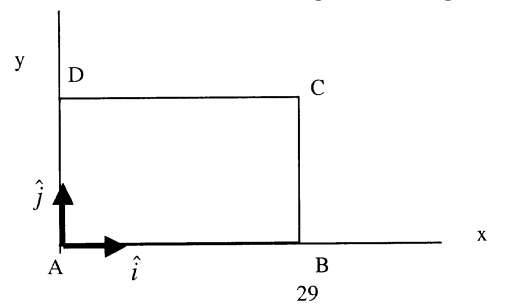

Escribe o representa en función de sus componentes cada uno de los siguientes vectores

$$
A \vec{B} ; A \vec{D} ; A \vec{B}+A \vec{D} ; A \vec{D}-A \vec{B}
$$

- Explica y plantea ¿Qué conceptos, operaciones y propiedades del álgebra vectorial aplicarías tú para determinar el ángulo que forman las diagonales AC y $\mathrm{BD}$ del rectángulo? (no es necesario que realices el cálculo exacto sólo explica tu planteamiento).

Explicación:

3. En el sistema de referencia adjunto, dibuja y representa mediante flechas cada uno de los siguientes vectores dibujándolos uno a continuación del otro.

$\vec{a}=2 \hat{i}+3 \hat{j} ; \vec{b}=4 \hat{i} ; \vec{c}=-2 \hat{i}-3 \hat{j} ; \vec{d}=-4 \hat{i}$

- ¿Cuánto valen las componentes rectangulares del vector $\vec{a}+\vec{b}$ ?

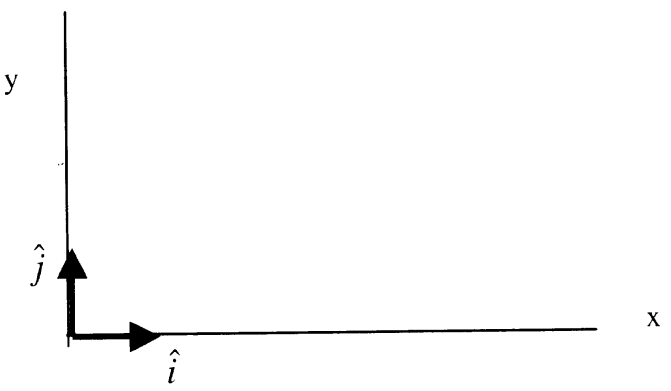

Explicación:

4. Dos partículas A y B se mueven en línea recta en sentido opuestos con MRUA y MRU respectivamente. En el instante $\mathrm{t}=0$ segundo, las posiciones de cada partícula sobre el eje $\mathrm{X}$ se muestran en la figura indicando las flechas el sentido de sus movimientos.

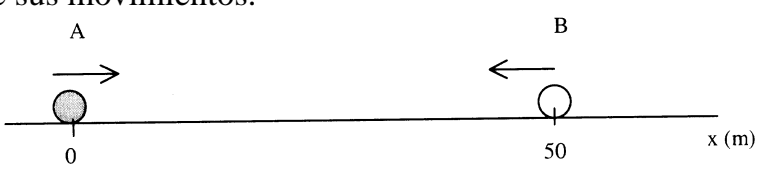

La posición de la partícula A en función del tiempo está representada por la ecuación

$$
x_{A}(t)=20 t-2 t^{2}
$$

y la posición de la partícula B en función del tiempo por el gráfico que se presenta a continuación.

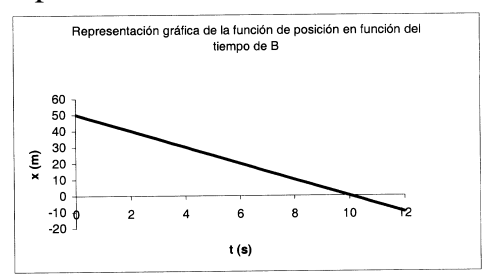

Escribe la ecuación que representa la función gráfica $x_{B}(t)$ de la partícula B.

Explicación:

- Representa gráficamente la posición en función del tiempo $x_{A}(t)$ de la partícula A.

- Plantea y explica ¿Cómo determinarías el instante y la posición en que las partículas A y B se cruzan?

Explicación:

5. Describe y explica dos situaciones cotidianas, una en que habitualmente se utilice el concepto de campo escalar por ejemplo, campos de temperatura, presión, densidad, etc. y otra en que se utiliza el concepto de campo vectorial como campo de velocidades, de fuerzas, eléctrico, magnético, gravitatorio.

Situación para Campo escalar:

Situación para Campo vectorial: 
6. ¿Cuáles de las siguientes magnitudes pueden ser descritas como campos?. Explica ¿Por qué? y fundamenta si son campos escalares o vectoriales.

- Dinero en un Banco

- Velocidad del agua en una corriente

- Temperatura en la superficie de un sartén

7. Juan y Pedro conversan acerca de ¿Cuál modelo matemático es mas apropiado para describir un fluido (líquido o gas) en movimiento?

\section{Juan dice :}

"Un fluido en movimiento es un medio continuo, pero podemos describir su movimiento considerándolo como un sistema o conjunto de muchas partículas en movimiento, es decir, imaginamos que dividimos el fluido en pequeños elementos de volumen que podemos llamar partículas de fluido y seguir el movimiento de cada una de estas partículas. Luego, para responder una pregunta como ¿Cuáles son los valores de la velocidad, densidad u otra magnitud en algún punto del fluido como una función del tiempo? Se puede responder si determinamos las coordenadas en función del tiempo de cada partícula, las cuales entonces, describirán el movimiento del fluido".

\section{Pedro afirma:}

"Es verdad, un fluído en movimiento es un medio contínuo que se mueve por una región del espacio. Pero, seguir la trayectoria y movimiento de las partículas individuales o de pequeñas gotas de líquido es un enorme trabajo, porque si consideramos un punto especifico del espacio del fluido y deseamos determinar la velocidad, densidad u otra magnitud, a medida que transurre el tiempo, debemos considerar que el fluido en ese punto está siendo reemplazado por fluido nuevo debido a su movimiento. Por lo tanto, para describir el movimiento de un fluido, lo mejor es enfocar nuestra atención en lo que está ocurriendo en cierto punto del espacio, en un determinado instante, más bien que en lo que está ocurriendo a una partícula determinada, es decir, lo más apropiado es describir el movimiento especificando la velocidad, la densidad u otra magnitud como una función de la posición y del tiempo".

¿Cuál modelo elegirías tú, el de Juan o el de Pedro? Explica y justifica tu elección.

Explicación:

8. En la figura se representan en algunos puntos del plano $\mathrm{XY}$, los vectores del campo de fuerzas escrito por la expresión

$$
\vec{F}=y \hat{i}+x \hat{j}
$$

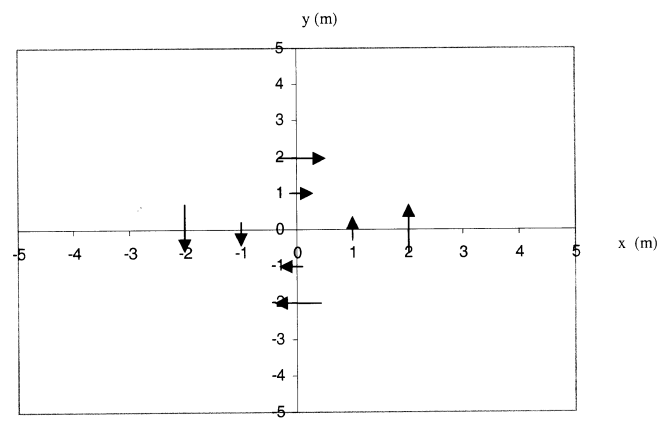

y en la Tabla se escriben para cada uno de estos puntos, las expresiones de los vectores de fuerza correspondientes.

\begin{tabular}{|l|l|l|}
\hline $\mathrm{x}(\mathrm{m})$ & $\mathrm{y}(\mathrm{m})$ & $\vec{F}=y \hat{i}+x \hat{j} \quad(N)$ \\
\hline 0 & 0 & 0 \\
\hline 0 & 1 & $\hat{i}$ \\
\hline 0 & 2 & $2 \hat{i}$ \\
\hline 0 & -1 & $-\hat{i}$ \\
\hline 0 & -2 & $-2 \hat{i}$ \\
\hline 1 & 0 & $\hat{j}$ \\
\hline 2 & 0 & $2 \hat{j}$ \\
\hline-1 & 0 & $-\hat{j}$ \\
\hline-2 & 0 & $-2 \hat{j}$ \\
\hline
\end{tabular}

- ¿Cuánto vale la fuerza en los puntos $(0,3)$; $(1,1)$; $(2,1) ;(2,2) ;(-2,2)$ y $(-2,-2)$ ?. Dibuja también estos vectores sobre la figura anterior.

Explicación:

- Imagina una hormiga que se mueve dentro de este campo de fuerzas siguiendo una trayectoria recta por la diagonal dada por la ecuación $y=x$. Elige dos puntos o posiciones de la hormiga en esta trayectoria y determina la fuerza que ejerce el campo sobre la hormiga en dichos puntos. Realiza también un dibujo o bosquejo que muestre la trayectoria con las fuerzas en las posiciones elegidas. (puedes utilizar la figura anterior)

Explicación:

9. Una superficie cúbica cerrada de lado a, que se muestra en la figura está colocada en una región donde hay un campo eléctrico $\vec{E}=c x \hat{i}$ paralelo al eje $\mathrm{X}$.

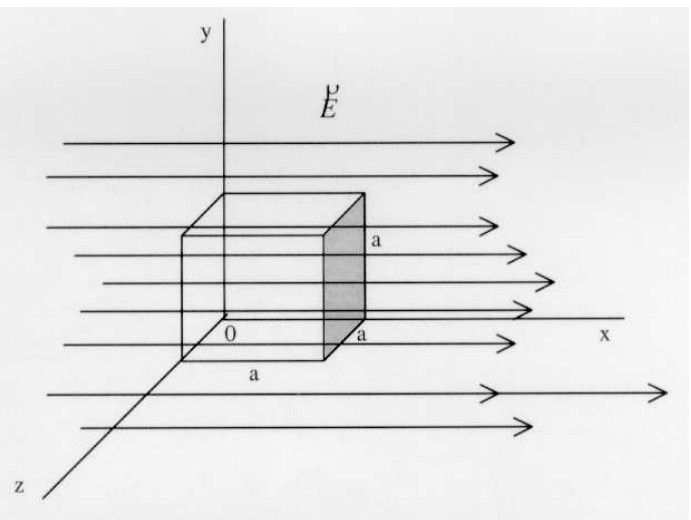


- ¿Cuánto vale el campo eléctrico en la cara sombreada de cubo?

Explicación:

- Explica y justifica mediante cálculos y/o argumentos que el flujo $\Phi$ del campo a través de la cara sombreada del cubo vale $\Phi=c a^{3}$

Explicación:
- Explica y justifica mediante cálculos y/o argumentos que la circulación $\mathrm{C}$ del campo a lo largo del contorno de la cara sombreada es nula (vale cero).

Explicación:

Si no respondes la pregunta, anota si es porque no conoces los conceptos de flujo y circulación.

10. Utiliza dibujos, ecuaciones y explicaciones para para dar una idea de lo entiendes por campo eléctrico. 